Revue québécoise de linguistique

\title{
Phénomènes atmosphériques dans le Dictionnaire explicatif et combinatoire du français moderne (DEC) : essai de description d'un champ lexical (neuf vocables du français)
}

\section{Suzanne Mantha et Igor Mel'čuk}

Volume 13, numéro 2, 1984

Grammaire et lexique

URI : https://id.erudit.org/iderudit/602521ar

DOI : https://doi.org/10.7202/602521ar

Aller au sommaire du numéro

Éditeur(s)

Université du Québec à Montréal

ISSN

0710-0167 (imprimé)

1705-4591 (numérique)

Découvrir la revue

Citer cet article

Mantha, S. \& Mel'čuk, I. (1984). Phénomènes atmosphériques dans le Dictionnaire explicatif et combinatoire du français moderne (DEC) : essai de description d'un champ lexical (neuf vocables du français). Revue québécoise de linguistique, 13(2), 271-323. https://doi.org/10.7202/602521ar 


\title{
PHÉNOMÈNES ATMOSPHÉRIQUES DANS LE DICTIONNAIRE EXPLICATIF ET COMBINATOIRE DU FRANÇAIS MODERNE (DEC): ESSAI DE DESCRIPTION D'UN CHAMP LEXICAL (NEUF VOCABLES DU FRANÇAIS)*
}

\author{
Suzanne Mantha et Igor Mel'čuk
}

Cet article se place dans une série de publications présentées par une petite équipe lexicographique rattachée au Département de linguistique de 1'Université de Montréal. Chacune d'elles se consacre au Dictionnaire explicatif et combinatoire (DEC) du français moderne: Mel'cuk et al. (1981), Elnitsky (1982), Mel’cuk (1982), ArbatchewskyJumarie et Iordanskaja (1982), Mel'cuk et al. (1983), Dagenais (à paraître), Cohen et Elnitsky (à paraître), Arbatchewsky-Jumarie et Iordanskaja (à paraître); une étude cumulative (Mel' cuk et al., (à parẫtre)) se trouve sous presse aux Presses de 1'Université de Montréal. Des détails importants et des références concernant le modèle linguistique sous-jacent au DEC sont donnés dans Mel'cuk (1981). Tout ceci nous évite la nécessité d'introduire ou de discuter les notions pertinentes, de fournir des explications ou de justifier l'approche adop-

* Le travail rapporté a été effectué dans le cadre du programme de recherches "Etudes lexico-sémantiques du français contemporain", subventionné par le Conseil de recherches en sciences humaines du Canada (subvention no 410-82-0549). 
tée. Nous présupposons, chez notre lecteur, des connaissances suffisantes du modèle et ne touchons pas aux problèmes gênéraux de base. Le but spécifique de cette publication est d'illustrer les méthodes et les considérations qui régissent la description lexicale dans un DEC et surtout d'analyser en profondeur quelques questions techniques relevant du domaine suivant: formulation des définitions. Nous nous efforçons surtout de démontrer la description d'un champ lexical, étant donnē qu'un des principes de base du DEC est 1'organisation de la description lexicographique par champ lexical. Nous refusons de traiter des lexèmes sēparés.

Notre texte sera organisé en trois parties majeures: d'abord nous donnons les explications nécessaires au lecteur, puis nous présentons les neuf vocables "atmosphériques" ${ }^{1}$ et enfin, nous en abordons la discussion, en utilisant le matériel lexicographique cité.

1. Traits les plus saillants du DEC et symboles utilisês

Pour faciliter la tâche de notre lecteur, nous nous permettons d'indiquer les traits les plus saillants du DEC et de donner une description sommaire des symboles utilisés surtout pour les fonctions lexicales dont la comprêhension serait difficile.

1. Nous avons préféré 'atmosphêrique' à 'météorologique' (qu'on voit dans certains dictionnaires) puisque 'météorologique.' semble luimême être sémantiquement dérivé de 'atmosphère/atmosphérique ': météorologique = 'qui concerne la météorologie' météorologie = 'étude scientifique des phénomènes atmosphériques'

atmosphérique = 'qui concerne I'atmosphère'

atmosphère = 'couche d'air qui entoure le globe terrestre' 
1.1 À la différence de la plupart des dictionnaires existants, le DEC se veut partie intégrante d'un modèle linguistique complet. Notre dictionnaire vise surtout les objectifs d'une approche scientifique et laisse de côté les considérations pragmatiques, commerciales et même pédagogiques. Joint à une grammaire formelle de la langue (dans notre cas, le français), le DEC doit assurer les correspondances entre une représentation sémantique et toutes les représentations syntaxiques profondes porteuses du même sens. Par conséquent, d'une part, le DEC doit contenir, pour tout lexème individuel, toutes les informations pertinentes nécessaires pour la donnée desdites correspondances; d'autre part, ces informations doivent être présentées de façon formalisée.

1.2 Le DEC est élaboré au sein d'une théorie linguistique particulière: la théorie Sens-Texte. Le formalisme et les notions de base y sont intimement liés.

1.3 L'unité de description dans le DEC est un Zexème, c'est-à-dire un mot ou une locution idiomatique pris dans une seule acception. Chaque lexème fait l'objet d'un article de dictionnaire et chaque article de dictionnaire ne décrit qu'un lexème. Les lexèmes apparentés sont regroupés dans un vocable. Un trait important de notre DEC est qu'il traite également les lexèmes et les expressions idiomatiques multilexémiques telles que donner sa langue au chat, de façon a, de neige, etc.; ces expressions apparaissent comme des vedettes suivies d'articles de dictionnaire.

Pour distinguer les lexèmes, nous utilisons un système de numérotation établissant leur hiérarchie sêmantique. Nous utilisons: 
- les exposants pour les homonymes (par exemple, vol ${ }^{1}$ [vol d'oiseau] et vol ${ }^{2}$ [vol à main armée].

- les chiffres romains si la différence sémantique entre les lexèmes d'un même vocable est suffisamment grande et a un caractère irrégulier (par exemple, GRELLE I et GRÊEE II).

- les chiffres arabes si la différence sémantique entre les lexèmes $n^{\prime}$ est pas tellement importante et a un caractère plutôt régu1ier (par exemple, PLUIE II.1 et PLUIE II.2).

- les lettres de l'alphabet latin si la différence sémantique est minime et tout à fait régulière.

1.4 Dans le DEC, 1'article de dictionnaire a une structure formelle rigide; il est composé de trois zones principales, subdivisées en souszones (dont la caractérisation ne sera pas exhaustive ici)

\subsubsection{La zone sémantique ou DÉFINITION}

Pour un lexème à sens prédicatif (au sens logico-sémantique), la définition est toujours construite à partir d'une forme propositionnel1e, qui est une expression composée du lexème en question et des variables correspondant à tous les participants (= actants sémantiques) de la situation décrite par le lexème. Par exemple, définir PLUIE II.2 (pluie d'insultes <d'obus>) revient à dêfinir 1'expression pluie de $X$. N.B.: Le langage des définitions, étant un métalangage par rapport au français, peut quelquefois faire entorse aux normes stylistiques du français êcrit. 


\subsubsection{La zone syntaxique ou SCHÉMA DE RÉGIME}

Le schéma de régíme (SR) est un tableau qui spécifie, pour chaque actant sémantique du lexème vedette, 1'actant syntaxique profond correspondant et énumère tous les moyens de surface qui peuvent servir à I'expression de ce dernier. On y indique quels actants sont employés de façon obligatoire. Si un actant sémantique ne peut être réalisé au niveau de surface, la colonne qui lui correspond n'est pas remplie et on y met un trait. Le SR est suivi d'exemples illustrant la combinaison des actants avec le lexème vedette.

Certains lexèmes peuvent avoir plusieurs variantes du SR, appelées modifications, lesquelles se distinguent par le nombre ou l'ordre des actants syntaxiques. La règle de correspondance entre les modifications a la forme suivante (dans notre exemple, nous montrons la correspondance entre une modification à deux valences et une autre qui en a trois):

Mod 1

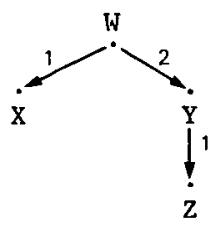

$\operatorname{Mod} 2$

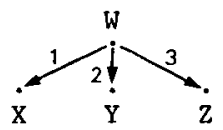

La règle de correspondance est illustrêe par un exemple: exemple pour Mod $\longrightarrow \longrightarrow$ exemple pour Mod 2

\subsubsection{La zone lexico-combinatoire ou FONCTIONS LEXICALES}

Les fonctions lexicales (FL) présentent l'ensemble de la cooccurrence lexicale restreinte intéressant le lexème considérê. Elles permettent de décrire de façon systématique un ensemble de locutions plus ou moins figées. Une FL est, grossièrement parlant, un sens assez abs- 
trait tel que son expression linguistịque dépend du lexème auquel cette FL vient se joindre. Ce lexème est dit mot clé, ou argument, de la FL. L'écriture standard d'une FL est de la forme suivante:

$$
f(X)=Y \text {. }
$$

où $f$ est la FL, $X$ son argument et $Y$, la valeur de 1a FL $f$ pour cet argument, c'est-à-dire l'ensemble des expressions linguistiques exprimant le sens ou le rôle syntaxique donné. Cependant, pour des raisons d'économie d'espace, le DEC emploie une écriture simplifiée: dans 1 'entrée du lexème $X$ on écrit simplement

$$
\text { f : } Y
$$

(vu que 1 'argument $X$ reste le même pour toute $f$ dans un article donné - c'est toujours le lexème vedette).

\section{LISTE DES FONCTIONS LEXICALES}

La liste ci-dessous ne répertorie que les FL nécessaires aux articles de dictionnaire présentés plus loin.

1. Syn, Syn ${ }_{c}, \operatorname{Syn}_{\supset}$, Syn $_{n}$ : synonymes et quasi-synonymes, ou syno-

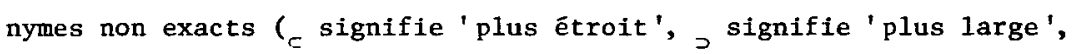
tandis que $n$ dénote une intersection de sens).

$$
\begin{aligned}
& \text { Syn }(\text { espoir })=\text { espérance } \\
& \text { Syn }(\text { pluie } I)=\text { averse } I \text {; bruine; crachin; ondée } \\
& \text { Syn }_{\supset}(\text { pleuvoir } I I .1)=\text { couler, gicler } \\
& \text { Syn }_{n}(\text { pluie } I)=\text { mauvais temps }
\end{aligned}
$$

2. $\mathrm{Conv}_{i j \mathrm{k}}$ : conversif, ou un lexème qui dénote une relation converse de la relation exprimée par le mot clé de cette FL; les indices montrent 1 'ordre des actants syntaxiques associés au conversif relati- 
vement à l'ordre de base (l'ordre des actants associés au mot clé du conversif), qui est toujours "123". Par exemple, $C_{312}\left(C_{0}\right)$ veut dire que les troisième, premier et deuxième actants du mot clê $\mathrm{C}_{0}$ deviennent respectivement les premier, deuxième et troisième actants de Conv.

Conv $_{21}$ (craindre) $=$ effrayer

3. Anti, Anti ${ }_{C}$, Anti $i_{J}$, Anti $_{n}:$ antonymes et quasi-antonymes.

Anti (respect) $=$ irrespect

Anti $_{n}($ aider $)=$ gêner

Anti se combine facilement avec d'autres FL pour former des FL complexes (voir plus bas).

4. Gener: mot générique dont la combinaison avec un dérivé syntaxique (voir le numéro 6 plus bas) du mot clé est synonyme du mot clé.

Gener (pluie I) = précipitation ([de pluie ]); phénomène atmosphérique

5. Figur: métaphore codifiée par la langue dont la combinaison avec le mot clé est un synonyme (plus étroit) du mot clé.

Figur (neige $I)=$ tapis, manteau [de neige]

6. $S_{0}, V_{0}, A_{0}, A_{d v}$ : dérivés syntaxiques - nom, verbe, adjectif, adverbe.

Par "dérivé syntaxique d'un lexème $C_{0}$ ", on entend un autre lexème ou un syntagme qui a le même sens que $\mathrm{C}_{0}$, mais qui appartient à une autre partie du discours. Soulignons qu'il s'agit ici des parties du discours au niveau syntaxique profond, si bien que nous entendons par $\mathrm{Adv}_{0}$ tous les mots et syntagmes invariables du français (y inclus les 
prépositions, les conjonctions, etc.) dont le comportement syntaxique est semblable à celui des adverbes.

$\mathrm{S}_{0}($ neiger $)=$ neige

$\mathrm{V}_{0}$ (pluie II.I) = pleuvoir II.I

A $(g r e l e ~ I)=d e$ [grêle] [averse inuages de grêle]

$\mathrm{Adv}_{0}$ (pluie II.1) = en [pluie]

7. S res : nom typique pour le circonstant du résultat.

$S_{\text {res }}($ neiger $)=$ enneigement

8. Sing: 'un quantum <une portion> régulièr(e) de...'

Sing $($ neige $)=$ flocon $[$ de neige]

Sing (grêle $I)=1 /$ grềlon

9. Mult: 'ensemble régulier de ...'

Mult $(a b e i z l e)=$ essaim

10. $A_{1}, A_{2}, A_{3}, \ldots$ : attribut typique pour le premier, deuxième actant du mot clé en question.

$A_{1}$ (mépris) $=$ plein, rempli [de mépris]

$\mathrm{A}_{2}$ (mépris) $=$ couvert $[$ de mépris $]$

11. Magn: 'très ', 'intense (ment)', 'à un degrê élevé'.

Magn $($ pluie $I)=$ grosse

Magn (neiger) $=$ abondinment, $d m$, fort

Les expressions de 1a Fl Magn peuvent différer quant au degré d'intensitê dêsigné, ce qui est indiqué à 1'aide du symbole < ('plus grand que '). La FL Magn peut être précisêe par un exposant sêmantique 
ou un indice actantiel. Une écriture comme Magn temp signifie qu'il s'agit d'une intensification temporelle.

Magn $^{\text {temp }}($ pluie $I)=$ grande $<$ continuelle, sans trêve

12. Epit: épithète standard sémantiquement vide (rêpétant une partie du sens du mot clé).

Epit (pluie II.1) = abondante

13. Plus, Minus: 'plus', 'moins'; ces FL s'emploient que dans une combinaison avec d'autres FL.

IncepPredPlus (pluie $I$ ) = s'accroitre, augmenter, redoubler

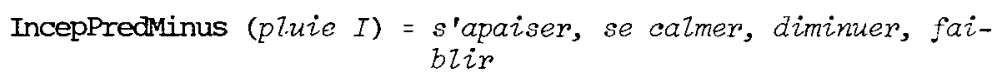

14. Bon: 'bon' - expression qu'on emploie comme une louange standard codifiée par la langue.

Bon (pluie $I)=$ bienfaisante

15. Loc in: préposition régissant le mot clé et signifiant une localisation, autrement dit la préposition qui signifie 'se trouvant dans'. $\operatorname{Ioc}_{\text {in }}($ pluie $I)=$ sous [la pluie]

16. Pred: 'prêdicat' - verbalisateur des FL adjectives: par exemple, pour décrire le sens 'être moins que' ou 'devenir moins que', on utilise 1'écriture PredMinus ou IncepPredMimus. Cette FL n'apparaît qu'en combinaison avec d'autres FL. (Voir les exemples donnés pour la FL Mimus.)

17. Oper $_{1}$, oper $_{2}, \ldots$ : verbe sémantiquement vide qui prend le nom du premier, du deuxième, $\ldots$ actant de la situation $C_{0}$ comme son sujet 
grammatical (SG) et le mot clé $C_{0}$ comme son complément d'objet (CO) principal.

Cette formulation ne đoit pas être interprētée dans le sens suivant: tout verbe remplissant le rôle d'un oper ${ }_{1}$, oper 2 , etc. serait toujours sémantiquement vide, c'est-à-dire qu'il serait complètement dépourvu de sens. Parmi les oper ${ }_{1}$, oper $_{2}$, etc., on rencontre, bien sûr, des verbes vides mais aussi des verbes semi-vides (d'une signification très abstraite et vague) et des verbes sémantiquement pleins. Les verbes de ces deux derniers types ne deviennent vides qu'accouplés à leurs mots clés; dans ce cas-1à, le contenu sêmantique d'un tel verbe est inclus dans celui de son mot clé et le choix du verbe est imposê automatiquement.

La même réserve s'applique aux FL Func ${ }_{i}$ et Labor $_{i j}$ (voir plus bas).

$$
\begin{aligned}
& \text { oper }_{1}(\text { attention })=\text { faire } \\
& \text { oper }_{2}(\text { attention })=\text { attirer } \\
& \text { oper }_{2}(\text { pluie II.2) = recevoir [une pluie de compliments] }
\end{aligned}
$$

18. Func $c_{0}$, Func $_{1}$, Func $_{2}, \ldots$ : verbe sëmantiquement vide qui prend $\mathrm{C}_{0}$ comme son SG et, au cas où la situation $\mathrm{C}_{0}$ a des actants, le nom $\mathrm{du}$ premier, du deuxième, ... actant de $c_{0}$ comme son co principal.

$$
\begin{aligned}
& \text { Func }_{0}(\text { pluie I) = tomber } \\
& \text { Func }_{1} \text { (aide) = venir, provenir [de quelqu'un] } \\
& \text { Func }_{2} \text { (pluie II.2) = tomber [sur quelqu'un/quelque chose] }
\end{aligned}
$$

19. Labor ${ }_{i j}$ : verbe sêmantiquement vide qui prend le nom de l'actant $i$ comme son SG, le nom de l'actant $j$ comme son Co principal, et $\mathrm{C}_{0}$ comme son deuxième $\mathrm{C} 0$. 


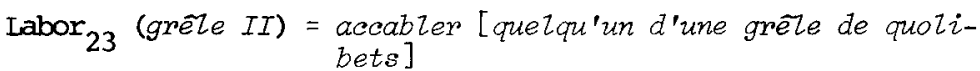

20. Involv: verbe qui prend $\mathrm{C}_{0}$ come son $S G$ et le nom signifiant l'objet qui subit 1 'action de la situation $C_{0}$ sans en être un participant, comme son $C 0$ principal.

Involv (pluie I) $=$ tomber [sur quelqu'un/quelque chose $]$

21. Incep, Cont, Fin, Prox: ' commencer', 'continuer', 'cesser', 'être sur le point de'; ces FL s'emploient le plus souvent avec d'autres FL.

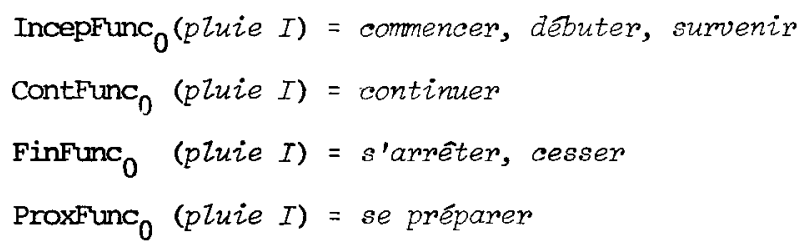

22. Perf: 'action complétée' (valeur de notre passê composé). Cette FL s'emploie le plus souvent en combinaison avec d'autres FL. $\mathrm{A}_{1}$ Perffunc ${ }_{0}$ récemment $($ neige $)=$ frầche, nouvelle

23. Caus: 'causer' ('faire en sorte que quelque chose ait lieu'). Cette FL s'emploie le plus souvent en combinaison avec d'autres FL.

$$
\begin{aligned}
\text { Caus, } \left._{2} \text { nonoper }_{2} \text { (grêle II }\right)= & \text { s'abriter, se cacher, se protéger } \\
& {[\text { de la pluie }] }
\end{aligned}
$$

En règle gênérale, celui qui cause une situation n'est pas un participant de cette situation; donc, le premier actant de Caus ne coïncide pas nécessairement avec un des actants du mot clé. Dans ce cas-là, la FL mentionnée ne comporte pas d'indices actantiels. Dans le cas contraire, la FL porte I'indice de l'actant correspondant. 
24. Son: 'émettre le son typique'.

Son $($ chien $)=$ aboyer

$\mathrm{S}_{0} \mathrm{Son}$ (chien) = aboiement

$\mathrm{S}_{0}$ Son $($ pluie I) = bmit, litt bruissement, litt crépitement

Du point de vue sémantique, une FL porte, en règle générale, sur le sens de son mot clé, ce sens étant pris comme un tout. Cependant, dans certains cas, une FL peut porter sur une des composantes sémantiques du mot clé, c'est-à-dire du lexème vedette, plutôt que sur le lexème "en entier". La composante pertinente est donc mise en indice (entre guillements simples et crochets) auprès du symbole de cette FL. Par exemple, dans 1'article de PLUIE I, nous trouvons 1'écriture suivante:

$$
\begin{aligned}
& \operatorname{Magn}_{[\text {'chute'] }} \text { : brutale, forte, violente } \\
& \text { Magn'['eau'] : abondante < diluvienne, torrentielle }
\end{aligned}
$$

Cela veut dire que ces Magn intensifient spécifiquement les notions de la chute d'eau et de la quantité d'eau qui tombe puisque une pluie brutale = 'une pluie dont la chute est brutale' et que une pluie abondante = 'une pluie dont la quantité d'eau qui tombe est abondante'. (Les composantes 'chute' et 'eau' sont présentes dans notre définition de PLUIE I.)

Les FL énumérées jusqu'ici sont des FL simples. Nous avons déjà noté que certaines d'entre elles s'emploient assez régulièrement ou même exclusivement combinées à d'autres FL. Ces combinaisons sont des FL complexes. Le sens d'une FL complexe est obtenu à partir des FL simples qui la composent. Il faut cependant souligner qu'une FL com- 
plexe n'est pas une composition - au sens mathématique - des FL constituantes parce que sa valeur (= expression linguistique) ne s'obtient pas nécessairement par combinaison régulière des valeurs des FL constituantes. Voici quelques exemples:

$$
\begin{aligned}
& \text { Antimagn (neiger) }= \text { légèrement, peu, a peine } \\
& \mathrm{S}_{0} \text { Func }_{0} \text { (pluie I) }= \text { chute [de pluie]; précipitation ([de } \\
& \text { pluie]) } \\
& \text { nonoper }_{2} \text { (pluie II.2) = échapper [à une pluie d'obus] }
\end{aligned}
$$

Les relations syntactico-sémantiques entre les fonctions constituantes d'une FL complexe sont toujours déterminées par la syntaxe inhêrente de chaque FL simple (voir Mel'cuk, 1974b, p. 82-108), ce qui nous permet de ne pas marquer explicitement ces relations dans tous les cas. Par exemple, on écrira simplement CausFunc $c_{1}$ pour Caus $\stackrel{2}{\longrightarrow}$ Func $_{1}$ ou MagnManif pour Magn ATTR Manif. Cependant, si la FL complexe est telle que la relation entre les constituantes puisse échapper au lecteur, on emploiera l'écriture explicite avec la flèche syntaxique, comme par exemple:

Involv $\stackrel{2}{\longrightarrow} \mathrm{x}$ (pluie I) $=$ tomber [sur quetqu'un/quelque chose]

D'une FL complexe, qui exhibe des relations syntactico-sêmantiques directes entre les FL constituantes, il faut distinguer une configuration de FL où les FL ne sont pas liées entre elles, mais possèdent un argument (= un mot clé) commun, par exemple:

$$
\begin{aligned}
& \operatorname{Magn}_{[' \text { chute'] }}+\operatorname{Magn} \text { ['eau'] (pluie I) = battante } \\
& \text { Magn }_{[\text {'chute'] }}+\text { Func }_{0} \text { (pluie I) = s'abattre }
\end{aligned}
$$


Il reste une dernière remarque à faire à propos des FL. Jusqu'ici, il s'agissait des FL standard, c'est-à-dire des FL ayant des sens assez abstraits et des valeurs assez diverses. Mais il existe aussi des locutions figées qu'on ne peut pas décrire à 1'aide des FL standard parce que leurs sens sont trop particuliers. Dans ces caslà, nous utilisons des FL non standard présentées sous forme libre. Par exemple:

$\operatorname{Magn}_{[\text {'chute'] }}+$ tel qu'elle empêche de voir + Figur (pluie $\left.I\right)=$ rideau [de pluie] < mur [de pluie]

quand il y a beaucoup de (pluie I) = de [pluie] // pluvieux

\section{LISTE DES SYMBOLES ET ABRÉVIATIONS}

: partie du mot clé $[=1$ lexème vedette $]$ non soumise à une modification orthographique

ART

: le mot clé s'emploie avec un article (ou son êquivalent) selon les règles de la grammaire

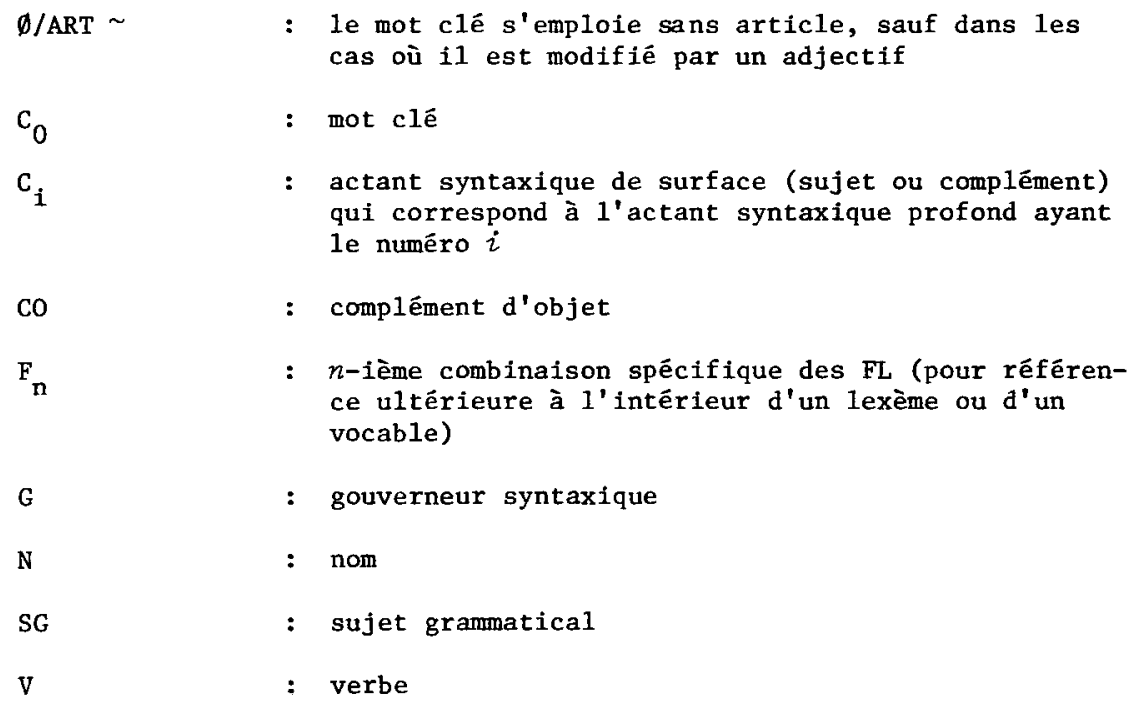

: actant syntaxique de surface (sujet ou complément) qui correspond à l'actant syntaxique profond ayant le numéro $i$

: complément d'objet

: n-ième combinaison spécifique des FL (pour référence ultérieure à l'intérieur d'un lexème ou d'un vocable)

: gouverneur syntaxique

: nom

: sujet grammatical

: verbe 
$(\mathrm{X})$

Mod

$\mathrm{Y}<\mathrm{X}>$

$[\mathrm{X}]$

$\Gamma \mathrm{x} \ldots \mathrm{Y}\urcorner$

ix

$/ / \mathrm{X}$
: X est facultatif

: modification syntaxique

: X est une variante de $Y$ (par exemple, la phrase II pleut sur la vilie <les promeneurs se lit II pleut sur la ville et Il pleut sur les promeneurs)

: 1) $X$ est un régime réduit d'une valeur d'une FL

2) $X$ est un commentaire

3) X est la composante sémantique du type ' comme si...'

: un groupe de lexèmes qui constitue un phrasème

: $X$ sont des conditions portant sur tout ce qui se trouve à gauche de | jusqu'à un point-virgule

: X est une expression fusionnée d'une FL, c'est-àdire que cette expression recouvre le sens du mot clé et de ce fait ne peut pas apparaître conjointement avec celui-ci. Par exemple:

Antimagn ['grains '] (grêle $I$ ) = menue

[grêle menue] // grésiz = 'grêle menue'

[*grésil (de) grête]

i $x$

: voir $\mathrm{X}[$ renvoi à $\mathrm{X}]$

: précède les phrasèmes [= locutions figées] comprenant le mot clê et ayant une composante sémantique commune avec ce dernier

: précède les phrasèmes comprenant le mot clê mais n'ayant aucune composante sémantique commune avec ce dernier

litt, spéc, rare: marques d'usage des expressions: littéraire, spécial et rare. Les marques d'usage ne caractérisent qu'une seule expression à la fois et sont placées devant cette expression

: précède les expressions spécifiquement québécoises. Ce symbole caractérise toutes ces expressions à la fois et est placé devant elles une seule fois 
2. Neuf vocables français: PLUIE, PLEUVOIR, NEIGE, NEIGER, DE NEIGE, GRÊLE, GRÊLER, GRÉSIL, GRÊLON

Nous avons choisi ces vocables pour tester nos méthodes lexicographiques sur la base d'un microsystème lexical.

2.1 Intuitivement, i1 semble que PLUIE : PLEUVOIR $\cong$ NEIGE : NEIGER $\cong$ GRÊLE : GRÊLER; par conséquent, notre description lexicographique doit refléter formellement cette proportionnalité perçue. Cependant, puisqu'on dit, par exemple, de nombreuses fluies mais pas *de nombreuses <cinq〉 neiges <grêles>, cette corrélation ne peut être qu'approximative, et ces différences d'usage doivent, elles aussi, trouver leur place dans les articles de dictionnaire correspondants.

2.2 GRÉSIL est une espèce de GRÊLE et GRÊLON en est un quantum (grê-

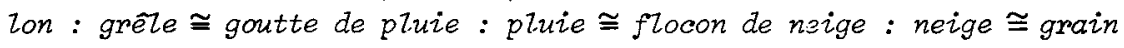
de riz : riz goutte d'eau : eau navire : marine ...); les trois entrées illustreront donc la description lexicographique des relations "genre espèce" et "substance $\sim$ quantum de cette substance" faite dans un DEC.

2.3 L'article DE NEIGE montre comment le DEC décrit les locutions figées de ce type, d'usage très restreint.

2.4 Nous touchons brièvement au problème de complexité sémantique relative aux noms et aux verbes "atmosphériques".

2.5 Un problème très spécifique se pose en ce qui a trait aux expressions régionales québécoises. Étant donné que le DEC est un dictionnaire du français standard, nous ne pouvons y admettre un vocabulaire provenant de plusieurs dialectes différents. Cependant, puisque cet 
article apparaît dans une publication québécoise et qu'un des auteurs est un locuteur natif de ce parler, nous avons décidé de rajouter, 1ã où nous le pouvions, des expressions québécoises. Comme une recherche appropriée n'a jamais été effectuée, nous ne pouvons garantir I'exhaustivité de la présentation. Pour éviter la confusion, toutes les expressions québécoises seront séparées des expressions standard françaises par un symbole spécial - une fleur de lis (voir plus haut). PLUIE, nom, fém.

I.

(a) Chute d'eau en tant que phénomène atmosphérique sous forme de gouttes, provenant des nuages I et tombant sur la terre ou $(\beta)$ cette eau qui tombe ... IIa pluie tombait ]

II.1. Chute abondante d'un liquide $X$ qui tombe en gouttes dispersées ... [une pluie de sang]

2. Chute ou vol ${ }^{1}$ de nombreux objets $x \ldots$ ou réalisation de nombreux actes $\mathrm{x} .$. . [une pluie de pierres et de coups]

I. ( $\alpha$ ) Chute d'eau en tant que phénomène atmosphérique sous forme de gouttes, provenant des nuages I et tombant sur la terre ou ( $\beta$ ) cette eau qui tombe $\left[=\mathrm{S}_{0}\right.$ (pleuvoir $\left.\left.I\right)\right]$.

\section{Connotations}

1) Quelque chose d'ennuyant ou de triste [voir certains phrasèmes dans PLUIE I]

2) Grande quantité, abondance [voir PLUIE II] 


\section{FONCTIONS LEXICAIES}

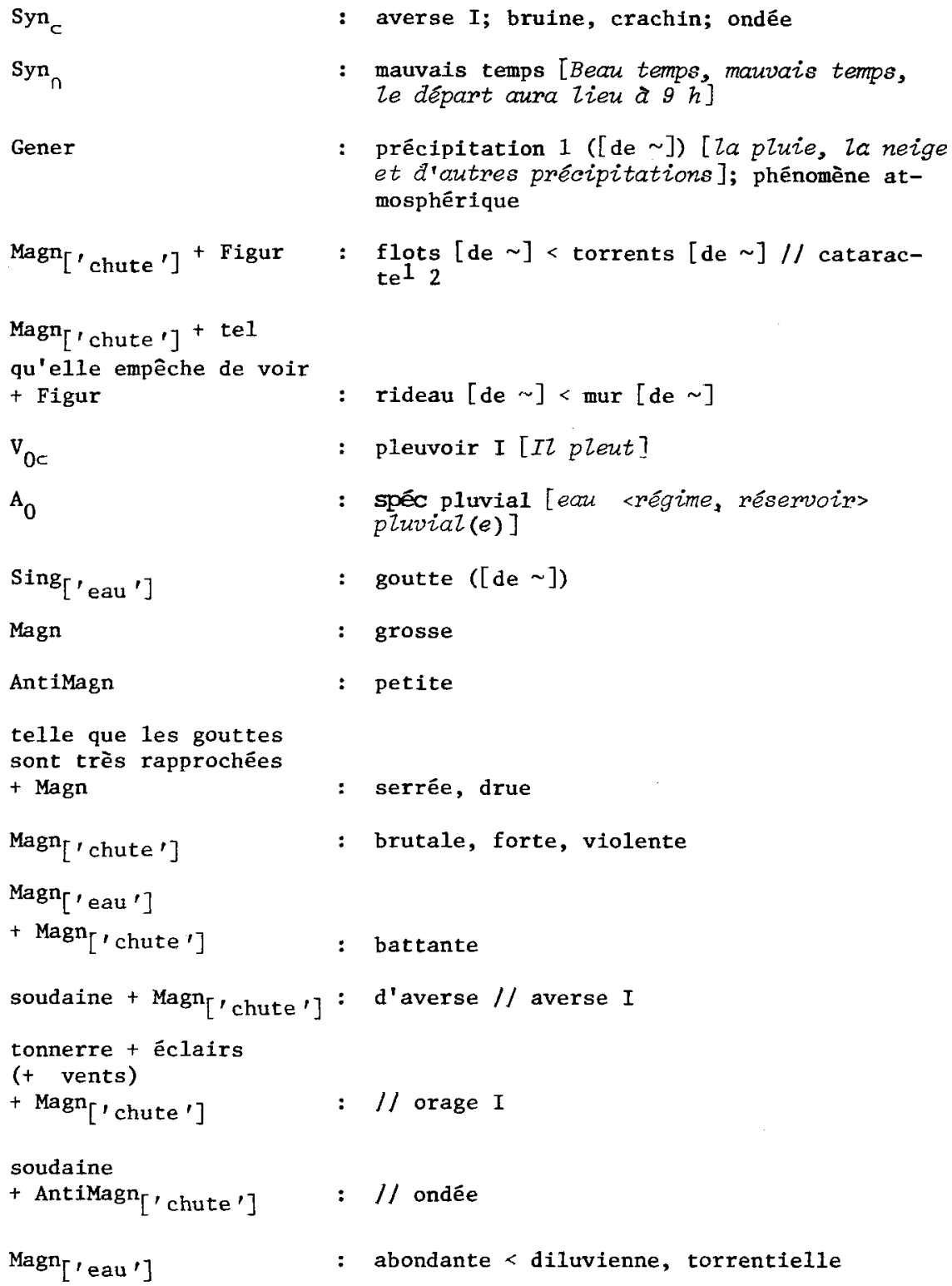




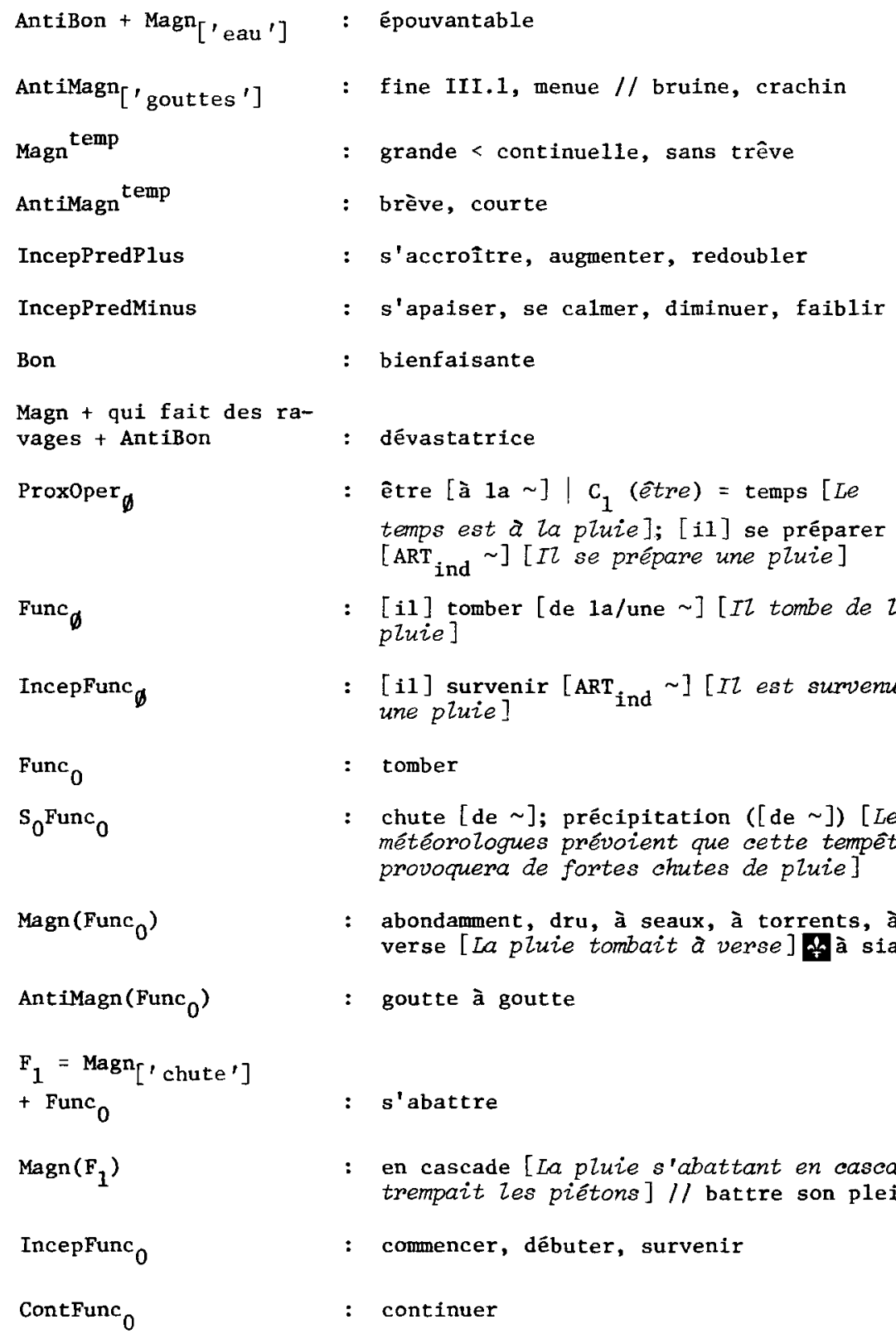




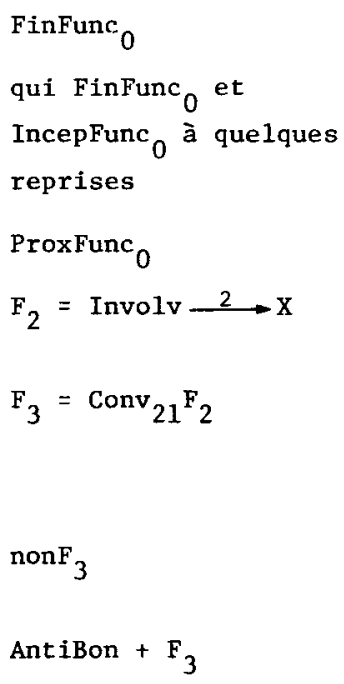

Son $+F_{2}$

Involv $\stackrel{2}{\longrightarrow}$ un être $X$ en causant que $X$ ait froid : refroidir $<$ glacer $[N=X]$

$\mathrm{F}_{4}+\operatorname{Involv} \stackrel{2}{\longrightarrow} \mathrm{X}$ en causant que $X$ soit mouillé

$\operatorname{MagnF}_{4}$

\section{Magn (transpercer,} tremper)
: s'arrêter, cesser

: intermittente

: se préparer [Une pluie se prépare]

: tomber $[\operatorname{sur} \mathrm{N}=\mathrm{X}$ ] [ La pluie tombait sur nos têtes]

: être [sous < dans> ART $\sim$, recevoir [ART ] [ La compagne est sous la pluie, Elze lâache son parapluie et reçoit la pluie]

: êchapper [à ART ] [Le voilier échappa à la pluie]

: essuyer [ART ] [Jean <Montréal> a essuyé une pluie]

: sous fै dans [ART ] [Ils gravirent la côte sous <dans> une pluie glaciale]

: s'abattre [sur $\mathrm{N}=\mathrm{X}$ ] [La pluie s'abattait (en cascade) sur la campagne <les promeneurs $>$; cingler, fouetter, frapper $[\mathrm{N}=\mathrm{X}]$ [La pluie fouettait les vitres lles piétons, nos jombes>]

: tambouriner [sur $\mathrm{N}=\mathrm{X}$ ] [ La pluie tambourinait sur les tentes]

: arroser, mouiller $[\mathrm{N}=\mathrm{X}]$

: laver $[\mathrm{N}=\mathrm{X}]$; tremper $[\mathrm{N}=\mathrm{X}]$; délaver \begin{tabular}{l|l}
{$[N=X]$; saucer $[N=X]$} & $X$ est une person- \\
ne; transpercer $[N=X]$ & $X$ est un vêtement
\end{tabular}

: jusqu'aux os 


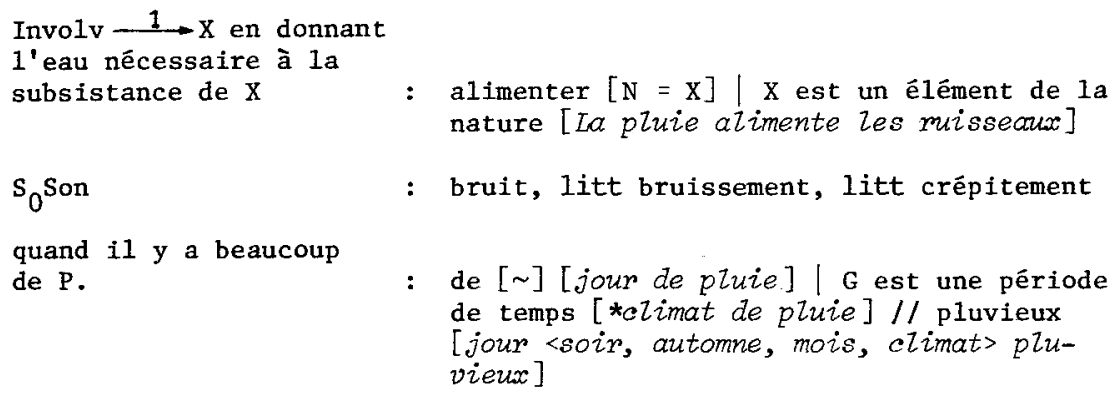

telle que P.[ $\beta]$ est froide

: froide $<$ glaciale

artéfact fabriqué pour protéger les personnes de 1a P.

: bottes $[$ de $\sim / /$ ciré, imperméable; parapluie

artéfact placé à la base

d'un toit pour permettre

I'écoulement des eaux

pluviales

: gouttière; gargouille

étude des mesures de $1 a$

quantité de $P$. tombée : pluviométrie 1

étude de la répartition

des P. sur le globe

: pluviométrie 2

instrument mesurant la

quantité de $P$. tombée en

un lieu, pendant un temps

déterminé

: spéc pluviomètre

temps où 11 n'y a pas

assez de $P$. (bien qu'il

doive $y$ en avoir)

: sécheresse

\section{EXEMPIES}

... une pluie si drue à ne pas se voir d'un bout du navire à I'autre

[P. Loti]. Il tombait une de ces pluies menues qui mouillent I'esprit autant que les habits, non pas une de ces pluies s'abattant en cascade, et jetant sous les portes cochères les passants essouflés, mais 
une de ces pluies si fines qu'on ne sent point les gouttes, une de ces pluies humides qui déposent sur vous d'imperceptibles gouttelettes et couvrent bientôt les habits d'une mousse pénétrante et glacée [G. de Maupassant]. Des pluies diluviennes et brèves s'abbattirent sur la ville [A. Camus]. ... comme s'il montrait quelque chose derrière le rideau mouvant de la pluie [A. Camus]. Content malgré la pluie qui tombait à verse, il reconduisit la jeune fille chez elle. On a installé de grands réservoirs pour recueillir la pluie. Dès que 1a pluie touchait le sol, elle se transformait en vapeur. Il secoua la pluie qui alourdissait son chapeau.

\section{d'après la connotation 1}

Après la pluie le beau temps = 'après la tristesse vient 1a joie' Ennuyeux comme la pluie = 'très ennuyeux' [ comme la pluie = $\operatorname{Magn}($ ennuyeux) ]

Faire la pluie et le beau temps = 'tout décider, mener tout' Ne pas être né <tombé> de la dernière pluie = 'avoir de 1'expérience' Parler de la pluie et du beau temps = 'dire des banalités'

Se mettre a l'eau par peur de la pluie = 's'avouer vaincu par peur de ne pouvoir affronter une situation' 
II.1. Pluie de $X=$ Chute abondante d'un liquide $\mathrm{X}$ qui tombe en gouttes dispersées [comme si c'était une pluie I].

RÉGIME

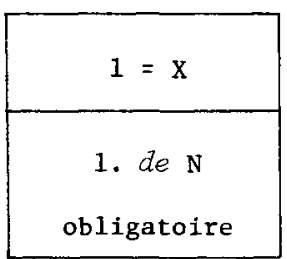

$\mathrm{C}_{1}$

: une pluie de sang

\section{FONCTIONS LEXICALES}

IncepPredPlus, IncepPredMinus, Func ${ }_{0}$, IncepFunc ${ }_{0}$, ContFunc ${ }_{0}$, FinFunc ${ }_{0}$ : $\uparrow$ PLUIE I

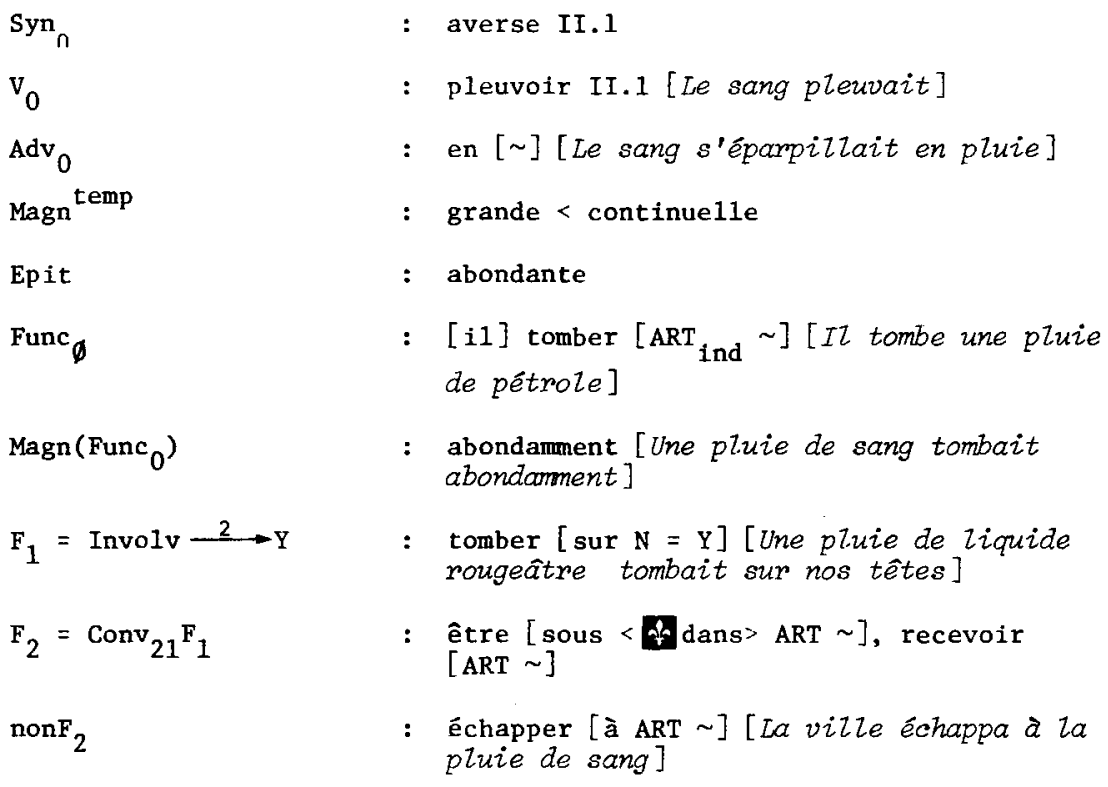


un être $\mathrm{X} \stackrel{1}{\longleftarrow}$ Causnonf $_{2}: \mathrm{s}^{\prime}$ abriter, se cacher, se protéger [de ART ]

$\mathrm{A}_{2}\left(\mathrm{~F}_{1}\right)=\mathrm{Loc}_{\text {in }} \quad:$ sous fons $[\mathrm{ART} \sim]$

$\operatorname{Magn}_{\left[{ }^{\prime} \text { chute' }\right]}+\mathrm{F}_{1} \quad: \quad \mathrm{s}^{\prime}$ abattre $[$ sur $\mathrm{N}=\mathrm{Y}]$

Son $+\mathrm{F}_{1} \quad:$ tambouriner $[\operatorname{sur} \mathrm{N}=\mathrm{Y}]$

\section{EXXMPLES}

Le sang s'éparpillait en pluie. Les arrosoirs répandent une pluie bienfaisante.

II.2. 1e plus souvent au sg. Pluie de $X[\operatorname{sur} Y]=$ Chute ou vol $^{1}$ de nombreux objets $X$ (qui tombent ou qui sont projetés sur $Y$ ) ou réalisation de nombreux actes (1iés de la façon la plus naturelle à) $X$ (qui concernent $Y$ ) tels que les intervalles de temps entre les $X$ sont minimes [comme si c'était une pluie $I][=$ FigurMult(X) $\mid$ X est un objet ou un acte].

RÉGIME

\begin{tabular}{|c|c|}
\hline $1=\mathrm{X}$ & $(2=\mathrm{Y})$ \\
\hline $\begin{array}{c}\text { 1. de } \mathrm{N}_{\mathrm{p} 1} \\
\text { obligatoire }\end{array}$ & \\
\hline
\end{tabular}

$\mathrm{C}_{1}$

: une pluie de projectiles <de coups, de cendre, d'insultes, de compliments> 


\section{FONCTIONS LEXICALES}

IncepPredP1us, IncepPredMinus, IncepFunc $\emptyset$, Func ${ }_{0}$, IncepFunc ${ }_{0}$, ContFunc $_{0}$, FinFunc ${ }_{0}: \uparrow$ PLUIE I

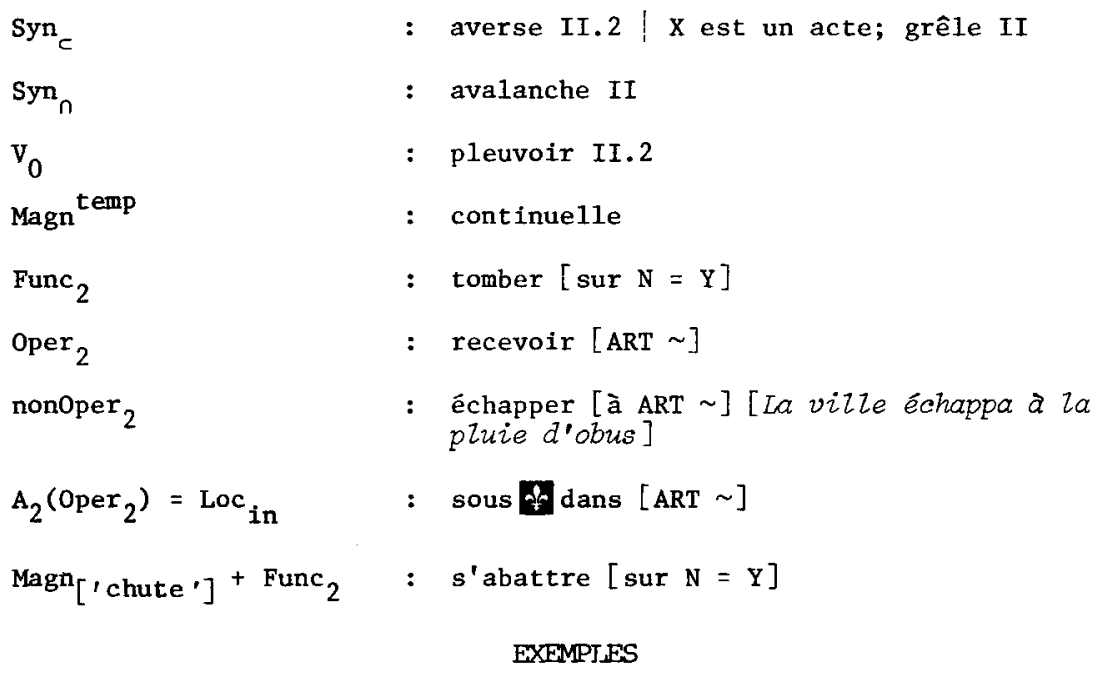

Une pluie de punitions s'abattit sur la classe. Durant 1'été, on peut assister à une pluie d'étoiles filantes. Les habitants ont fui la ville sous une pluie de pierres et de projectiles. Il recevra une gerbe d'éloges plutôt qu'une pluie de huées. PLEUVOIR, verbe.

I. La pluie I $(\alpha)$ a lieu [IL pleut]

II.1. Pluie II.1 de X lieu... [Le sang pleuvait]

2. Pluie II. 2 de $\mathrm{X}$ a lieu (sur $\mathrm{Y}$ )... [Les injures pleuvaient (sur eile)]

I. impers. Il pieut $=$ La pluie I $(\alpha)$ a lieu. 


\section{Connotation}

Grande quantitê, abondance [voir PLEUVOIR II]

\section{FONCTIONS LEXICALES}

$\mathrm{S}_{0 \mathrm{P}}$

AntiMagn ['eau']

$\operatorname{Magn}_{[\text {'gouttes'] }}$

AntiMagn $[$ 'gouttes']

Magn temp

$\mathrm{F}_{1}=$ Involv $\stackrel{2}{\longrightarrow} \mathrm{X}$

$$
\text { Magn }_{\text {[' eau '] }}
$$

: pluie I

: abondamment, fort, à flots, à seaux, à torrents, à verse, pop comme vache qui pisse, pop à boire debout, fam des cordes, 순 à siaux

: légèrement, à peine

: à grosses gouttes

: // pleuvasser, pleuviner, pleuvoter; bruiner; crachiner

: continuellement, sans arrêt

: [il] pleuvoir [ sur $\mathrm{N}=\mathrm{X}]$ [IZ pleut sur la ville <les promeneurs>]

\section{EXEMPLES}

I1 a plu trois jours de suite. Quand je suis sorti de la maison, il commençait à pleuvoir.

II.1. X pleut $=$ Pluie II.1 de X a lieu [ comme s'il pleuvait I]. RÉGIME

$\operatorname{Mod} 1$

$1=x$

1. $\mathrm{N}$

$\mathrm{C}_{1}$

: Le sang pleuvait 
Mod 2, impers

\begin{tabular}{|c|c|}
\hline 1 & $2=\mathrm{x}$ \\
\hline 1. i2 & $\begin{array}{c}\text { 1. ART } \text { part }_{\text {obligatoire }}^{\mathrm{N}} \\
\text { obligatis }\end{array}$ \\
\hline
\end{tabular}

$c_{1}+c_{2}$ : Il pleuvait du sang.

Mod 1

PLEUVOIR

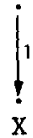

Le sang pleuvait
Mod 2, impers

PLEUVOIR

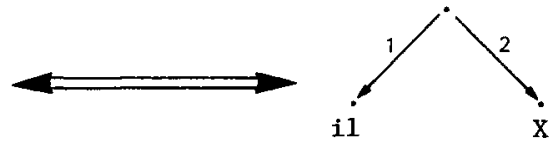

t pleuvait du sang

FONCTIONS IEXICALES

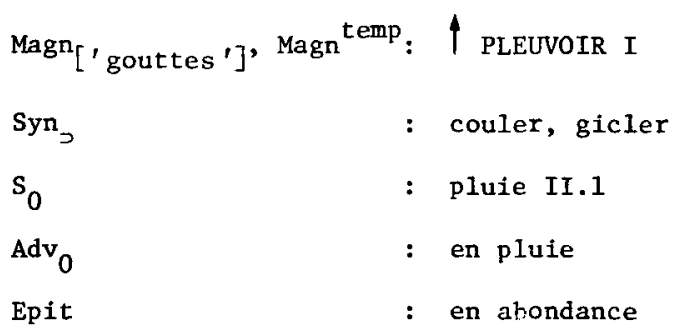

\section{EXFMPLES}

Des balles avaient troué les tonneaux et il pleuvait du vin sur nos têtes.

II.2. X pleut (sur $Y$ ) $=$ Pluie II.2 de X a lieu (sur Y) [come s'il pleuvait I] 
RÉGLME

Mod 1

\begin{tabular}{|c|c|}
\hline $1=\mathrm{X}$ & $(2=\mathrm{Y})$ \\
\hline $1 . \mathrm{N}_{\mathrm{pl}}$ & 1. $\operatorname{sur} \mathrm{N}$ \\
\hline
\end{tabular}

$c_{1}+\left(c_{2}\right)$

: Les boulets pleuvaient (sur la vilie)

Mod 2, impers

\begin{tabular}{|c|c|c|}
\hline$I$ & $2=x$ & $(3=\mathrm{Y})$ \\
\hline 1. iz & $\begin{array}{l}\text { 1. } \text { ART }_{\text {part }} \mathrm{N} \\
\text { obligatoire }\end{array}$ & 1. $\operatorname{sur} \mathrm{N}$ \\
\hline
\end{tabular}

$\mathrm{c}_{1}+\mathrm{c}_{2}+\left(\mathrm{C}_{3}\right) \quad$ : Il pleuvait des boulets (sur la vilze)

Mod 1

PLEUVOIR

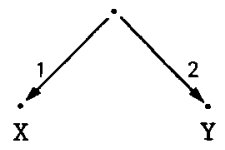

Mod 2, impers

PLEUVOIR

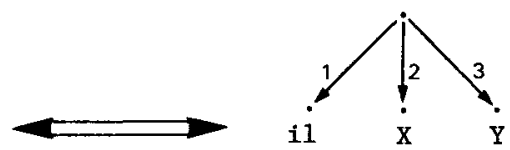

Les boulets pleuvaient (sur la vilze)

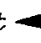

Il pleuvait des boulets (sur la vizle) 


\section{FONCTIONS LEXICALES}

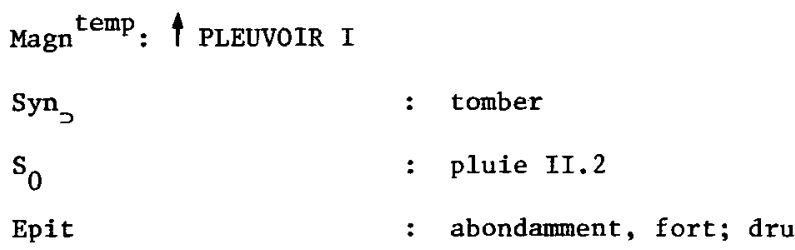

EXFMPLES

Les boulets pleuvaient [v. Hugo]. Ici pleuvent les nouvelles, vraies ou fausses [J. Michelet]. La raillerie pleut, drue comme mitraille [C. Beaudelaire]. Les branches brisées pleuvent autour de lui [H. de Montherlant]. Quanc les obus pleuvent, on ne pense plus à rien, au moins pendant quelques secondes [A. Adamov]. Il pleuvait des flèches de partout. Les biens, les dignités, les honneurs pleuvaient sur lui. Des petits fragments de son pare-brise, de ses phares et de sa calandre pleuvaient sur le sol dans un crépitement de confetti solides. C'est alors que du balcon les huées se sont mises à pleuvoir en direction du parterre.

Il en pleut de $X=I 1 \mathrm{y}$ en a beaucoup de $\mathrm{x}[\mathrm{Il}$ en pleut de ce genre de voitures ]

NEIGE, nom, fém.

(a) Substance blanche, peu dense, froide et légère formée d'eau congelée ou ( $\beta$ ) chute de cette substance provenant des nuages 1 et tombant sur la terre sous forme de flocons en tant que phénomène atmosphérique N. ( $\beta$ ) s'emploie seulement avec des FL ou avec des lexèmes capables d'actualiser ce sens. 


\section{Connotation}

Blancheur [voir certains phrasèmes]

\section{FONCTIONS LEXXICALES}

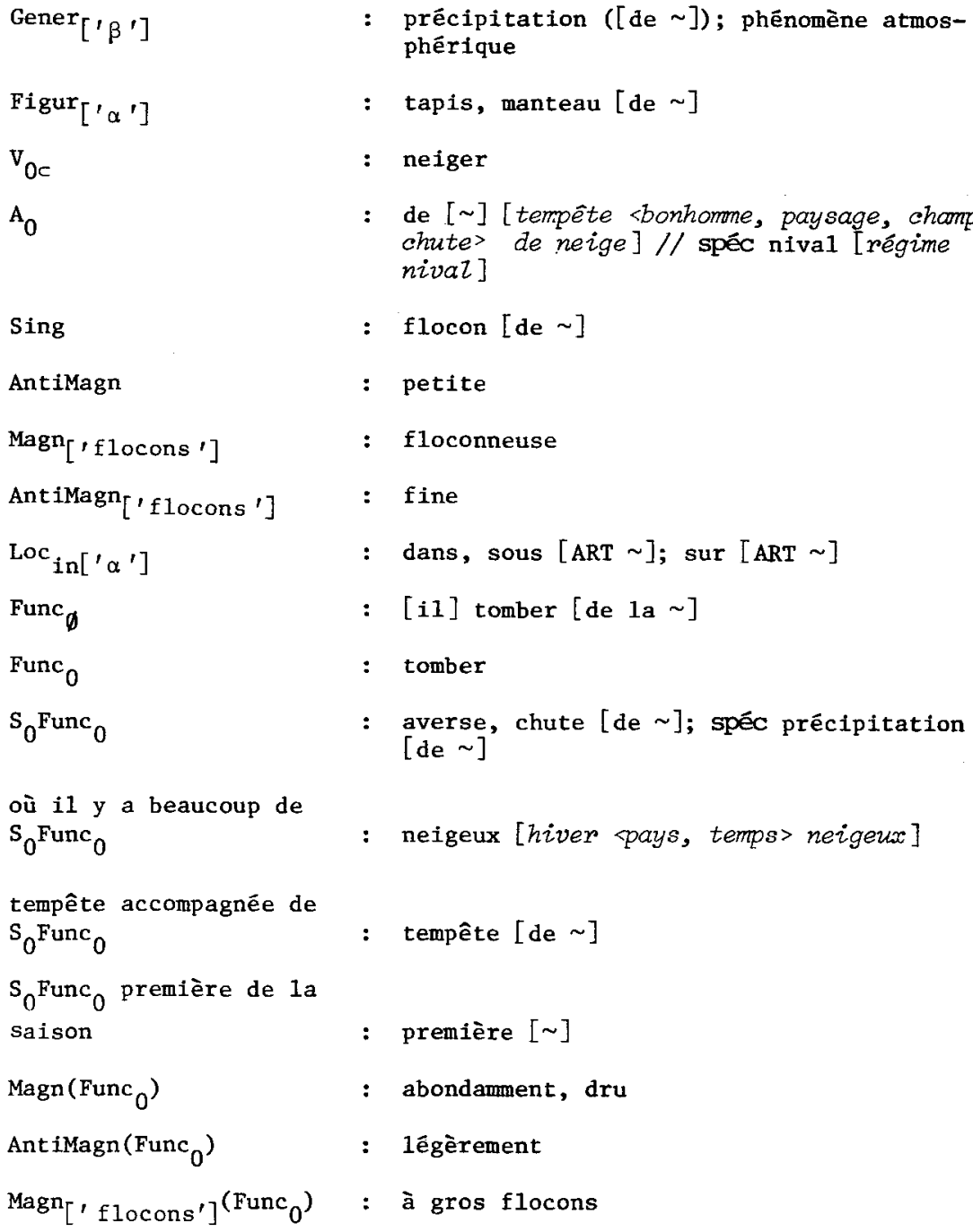




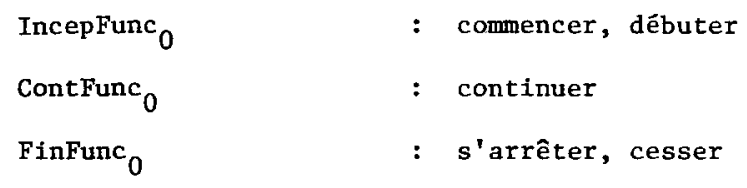

êpaisse couche de N. sur un objet dont le sommet est en pointe + ayant 1 'aspect de la ouate + $A_{2}$ Result $F_{1}$

: litt capitonné [de ] [somnet <arbre> capitonné de neige]

MagnResultF 1

: ensevelir, engloutir [N = X] | 1e plus souvent au passif: [sous ART ]

sèche

: sèche

Antisèche

: mouillée [Une neige mouizlée tombait en recouvrant les arbres]

quand on se déplace sur N. sèche, Son

: crisser [La neige arissait sous nos pas]

en se déplaçant, X s'enfonce dans la $\mathrm{N}$. à tel point qu'il lui est difficile de s'en sortir

: s'embourber, être pris, se prendre [dans ART ]

qui ne fond pas d'une année à 1 'autre

: éternelle(s), permanente(s), perpétuelle(s) | de préférence au plurie1

lourde

: lourde, pesante

Antilourde

: 1égère

qui est devenue dure

: durcte 
qui a la consistance de la poudre

N. folle soulevée par (des rafales de) vent

qui est formée de grains : granuleuse | $(\alpha)$

N. écrasée, sale et à moitié fondue

: // gadoue of sloche

accumulation de $\mathrm{N}$. fraichement tombẻe

fิ bordée [de ]

telle que 1a surface en est dure

: croûtée | ( $\alpha)$

masse de $N$. durcie en haute montagne et qui alimente parfois un

glacier

: // névé

masse de N. qui se détache d'une montagne et qui en dévale

: // avalanche

amas de $\mathrm{N}$. entassée par le vent

: Af banc $[$ de $\sim] / /$ congère

enlever la N. de W

: enlever, ôter $\left[\right.$ ART $_{\text {dêf }} \sim$ de $\left.\mathrm{N}=\mathrm{w}\right] / / \mathrm{dé}-$ neiger | (a) [On a déneigé le stationnement]

enlever la $\mathrm{N}$. de $\mathrm{W}$ avec une pelle

: // pelleter $\mid(\alpha)$ [On a pelzeté le chemin]

gros éperon d'acier muni de deux versoirs pour enlever la $\mathrm{N}$.

: chasse-neige 1

véhicule muni d'un chasse-neige 1 ou d'un dispositif hêlicoldal qui projette 1a N. à distance: chasse-neige $2 / /$ souffleuse

sport que $1^{\prime}$ on pratique sur la $\mathrm{N}$.

: $\quad$ sport de $\sim$ 
figure humaine façonnée grossièrement avec de la N.

: bonhomme de

équipement pour aller sur 1a $\mathrm{N}$.

: raquette; skis; traîneau, luge, traîne sauvage

vêhicule motorisé à une ou deux places avec skis à 1 'avant et sur chenilles

: motoneige

\section{EXXPMPLES}

Aucun bruit dans la forêt que le gémissement léger de la neige qui tombait sur les arbres. Elle tombait depuis midi; une petite neige qui poudrait les branches d'une mousse glacée, qui jetait sur les feuilles mortes des fourrés un léger toit d'argent, étendait par les chemins un immense tapis moelleux et blanc, et qui épaississait le silence illimité de cet océan d'arbres [G. de Maupassant]. La pluie s'était transformée en neige, une grosse neige floconneuse qui paraissait artificielle. Ils étaient encore à vingt milles de la mission quand la neige, qui tombait dru, ralentit leur allure. I1 marchait dans la neige jusqu'aux genoux. Le Nord-Est des États-Unis a été enseveli sous la neige. La neige avait débuté dimanche en soirée et a cessé hier dans l'aprèsmidi.

classe de neige

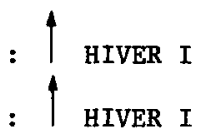

Train de neige

: HIVER I 
$\Gamma_{\text {De neige }}$

Oeufs en neige = Blancs d'oeufs battus jusqu'à ce qu'il soit blancs et fermes comme si c'était de la neige: 1 OEUF 1

Oeufs à Za neige = Blancs d'oeufs battus et pochēs, servis avec une crème: $\mid$ OEUF 1

NEIGER, verbe impers.

II neige = La neige tombe, cette chute étant un phénomène atmosphéricue.

\section{FONCTIONS L.EXICALES}

\begin{tabular}{|c|c|c|}
\hline $\mathrm{s}_{0}$ & : & neige \\
\hline$s_{\text {res }}$ & : & enneigement \\
\hline Magn & : & abondamment, dru, fort \\
\hline AntiMagn & : & légèrement, peu, à peine \\
\hline Involv $\stackrel{2}{\longrightarrow} x$ & : & 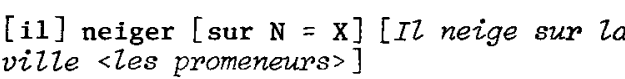 \\
\hline
\end{tabular}

\section{EXEMPLES}

Comme nous grimpions avec d'autres gens rencontrês en chemin, il commença à neiger fort... [E. LeRoy]. C'était au crépuscule, et il neigeait légèrement <peu>.

DE NEIGE, loc. adj.

litt. [X] de neige = [Une partie du corps ${ }^{1}$ II.I a X] d'une belle couleur blanche [teint, barbe, cheveu, peau, ..., mais pas *feuille <toiles de neige]. 


\section{FONCTIONS LEXICALES}

Syn $_{n} \quad$ : neigeux II [un duvet neigeux]

\section{EXEMPIES}

Elle avait un teint de neige ${ }^{2}$. Ses cheveux de neige adoucissaient ses traits.

GRÊLE, nom, fém.

I. ( $\alpha$ ) Substance composée de grêlons ou ( $\beta$ ) la chute de cette substance... [ La grềe commença à tomber]

II. Collectivité de nombreux objets X... ou de nombreux actes X... [une grêle de cailzoux <de questions>]

I. ( $\alpha$ ) Substance composée de grêlons ou ( $\beta$ ) chute de cette substance provenant des nuages I et tombant sur la terre en tant que phénomène atmosphérique | G. I( $\beta$ ) s'emploie seulement avec des FL et des lexèmes capables d'actualiser ce sens.

\section{Connotations}

1) Dommage [voir les phrasèmes et GRÊLE II]

2) Grande quantité, abondance [voir GRÊLE II]

2. Il faut insister sur le fait que les synonymes n'ont pas nécessairement la même combinatoire [duvet neigeux, *teint neigeux] et la combinatoire de chacun d'un groupe de synonymes doit être décrite dans son article de dictionnaire. Ainsi l'article de NEIGEUX II doit contenir une indication qui restreigne sa combinatoire à quelques expressions seulement: duvet 〈tulie, mache〉 neigeux $(s e)$. 


\section{FONCTIONS LEXICALES}

\begin{tabular}{|c|c|c|}
\hline $\operatorname{syn}_{c}$ & : & grésil \\
\hline $\operatorname{syn}_{n}$ & : & mauvais temps \\
\hline $\left.\operatorname{Gener}^{\prime} \beta^{\prime}\right]$ & : & $\begin{array}{l}\text { précipitation }([\text { de } \sim]) \text {; phénomêne atmos- } \\
\text { phérique }\end{array}$ \\
\hline $\mathrm{v}_{0 \subset}$ & : & grêler I \\
\hline $\mathrm{A}_{0}$ & : & de $[\sim]$ [averse 〈muage> de grêle] \\
\hline Sing & : & // grêlon \\
\hline $\operatorname{Magn}_{[}\left[\right.$chute' $\left.^{\prime}\right]$ & : & brutale, violente \\
\hline AntiMagn $_{[\text {'grains'] }}$ & : & fine III.1, menue // grésil \\
\hline IncepPredPlus & $:$ & s'accroître, augmenter, redoubler \\
\hline IncepPredMinus & $:$ & s'apaiser, se calmer, diminuer, faiblir \\
\hline Loc $_{\text {in }[' \beta ']}$ & : & sous dans $[$ ART $\sim]$ \\
\hline Func $\emptyset$ & $:$ & [ii] tomber [ de ART $\sim]$ \\
\hline Func $_{0}$ & $:$ & tomber \\
\hline $\mathrm{S}_{0}$ Func $_{0}$ & : & $\begin{array}{l}\text { averse, chute }[\text { de } \sim] \text {; spéc prêcipitation } \\
{[\text { de } \sim]}\end{array}$ \\
\hline $\begin{array}{l}\text { quand il y a beaucoup } \\
\text { de } s_{0} \text { Func } \\
0\end{array}$ & $:$ & rare grêleux [temps grêleux] \\
\hline IncepFunc $_{0}$ & : & commencer \\
\hline FinFunc $_{0}$ & : & s'arrêter, cesser \\
\hline $\operatorname{Magn}_{\left[{ }^{\prime} \text { chute'] }\right.}+$ Func $_{0}$ & : & s'abattre \\
\hline 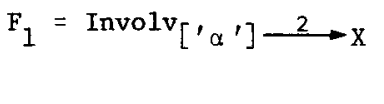 & : & $\begin{array}{l}\text { tomber }[\text { sur } \mathrm{N}=\mathrm{x}] \text { [La grêle tombait sur } \\
\text { la vizle] }\end{array}$ \\
\hline $\mathrm{F}_{2}=\operatorname{Conv}_{21} \mathrm{~F}_{1}$ & : & $\begin{array}{l}\text { être }[\text { sous }<\hat{f} \text { dans }>\text { ART } \sim] \text {, recevoir } \\
{[\text { ART } \sim]}\end{array}$ \\
\hline $\mathrm{nonF}_{2}$ & : & échapper [ã ART ] \\
\hline AntiBon $+\mathrm{F}_{2}$ & $:$ & essuyer $[$ ART ] \\
\hline
\end{tabular}


$\begin{aligned} \operatorname{Magn}_{[\text {'chute }]}+\mathrm{F}_{1} \quad: & \text { s'abattre }[\operatorname{sur} \mathrm{N}=\mathrm{X}] \text {; cingler, fouetter, } \\ & \text { frapper }[\mathrm{N}=\mathrm{X}][\text { La grêle cinglait <frap- } \\ & \text { pait> les vitres <les piétons>] }\end{aligned}$

\section{EXXMPLES}

Seule la frange de la tempête accompagnée de pluie, de grêle et de vents a frappé le sud du Mexique. C'est la grêle qui tombe. Tout à coup, une grêle brutale frappa le béton de plein fouet. La grêle a ravagé les récoltes. Il y a de la grêle sur les trottoirs. Il fut contraint de baisser la vitre et de passer la tête au dehors dans la pluie gelêe qui cinglait comme la grêle.

Méchant conme grêle

Tomber du conme grête

II. le plus souvent au sg. Grêle de $X=$ Ensemble de nombreux objets $X$ qui tombent ou sont projetés vigoureusement sur $Y$ ou de nombreux actes (liés de la façon la plus naturelle à) X qui concernent $\mathrm{Y}$ tels que les $\mathrm{X}$ sont nuisibles à $\mathrm{Y}$ et que les intervalles de temps entre ces $\mathrm{X}$ sont minimes [comme si c'était une grêle I] [ = AntiBon + FigurMult $(\mathrm{X}) \mid \mathrm{X}$ est un objet ou un acte].

RÉGIME

\begin{tabular}{|c|c|}
\hline $1=\mathrm{x}$ & $2=\mathrm{Y}$ \\
\hline 1. de $\mathrm{N}_{\mathrm{p} 1}$ & \\
obligatoire & \\
\hline
\end{tabular}

$\mathrm{C}_{1}$

: une grêle de cailloux <balles, injures, questions> 


\section{FONCTIONS IEXICALES}

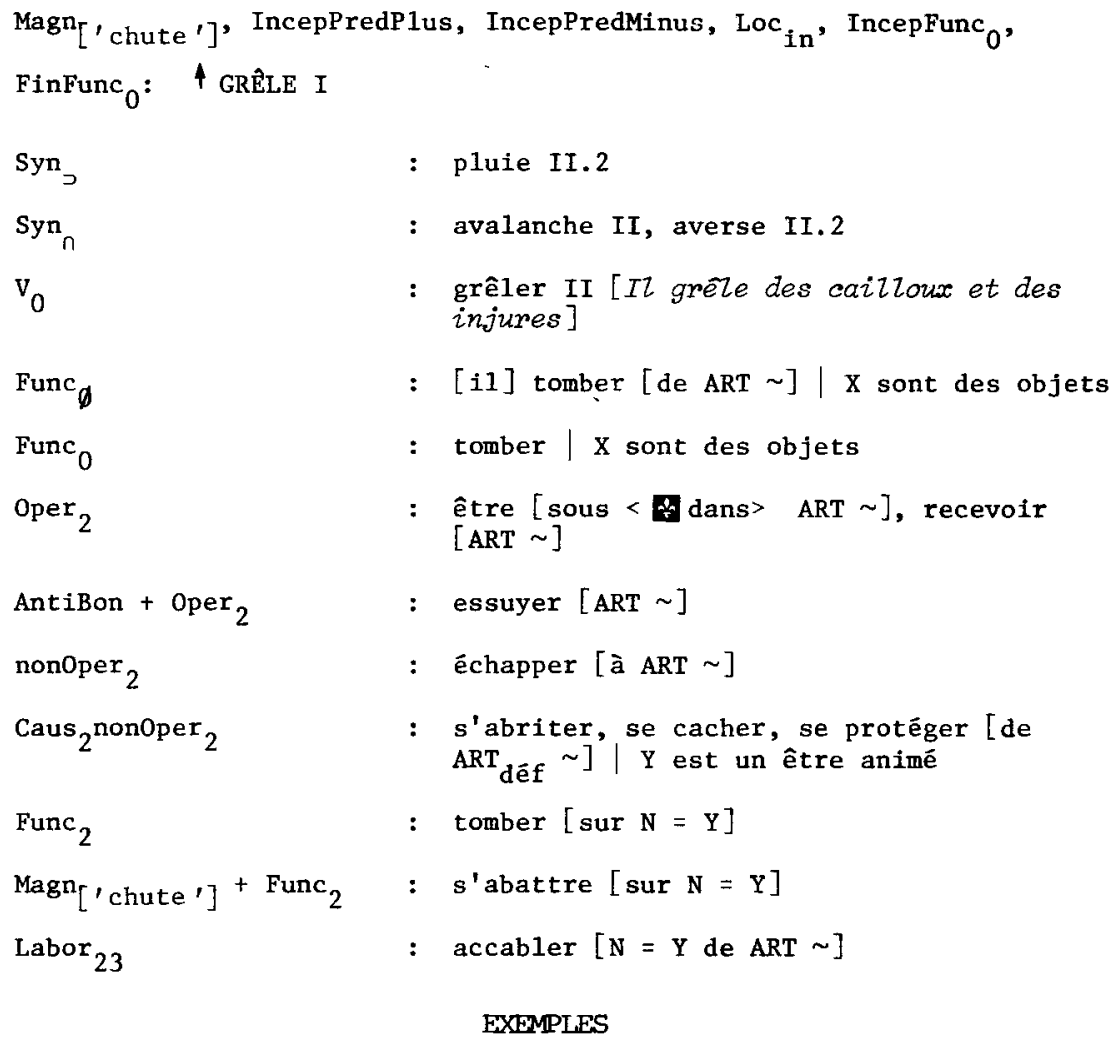

J'éviterai par une prompte fuite une grêle de coups qui seraient tombês sur moi [A.R. Lesage]. Il baissait le dos, attendant que la grêle habituelle des quolibets fut passée [R. Rolland]. Il croyait triompher de sa femme et 1'accablait alors d'une grêle de phrases qui répétaient la même chose et ressemblaient à des coups de hache rendant le même son [H. de Balzac]. Depuis un moment, la grêle de projectiles augmentait. 
GRËLER, verbe impers .

IZ grểe = La grêle I tombe, cette chute étant un phénomène atmosphérique.

\section{FONCTIONS LEXICAIES}

$\mathrm{S}_{0 \supset}$

Magn

AntiMagn
: grêle I

: fort

: // rare grésiller

\section{EXEMPLES}

I1 grêlera fort demain.

GRÉSIL, nom, masc.

Une fine ${ }^{3}$ III.1 grêle I.

\section{FONCTIONS LEXICALES}

Syn $_{n}$, Gener, Sing, IncepPredPlus, IncepPredMinus, Loc ${ }_{\text {in }}$, Func $\phi$, Func $_{0}$, $\mathrm{S}_{0}$ Func $_{0}, \mathrm{~F}_{2}$, nonF 2 : † GRÊLE I

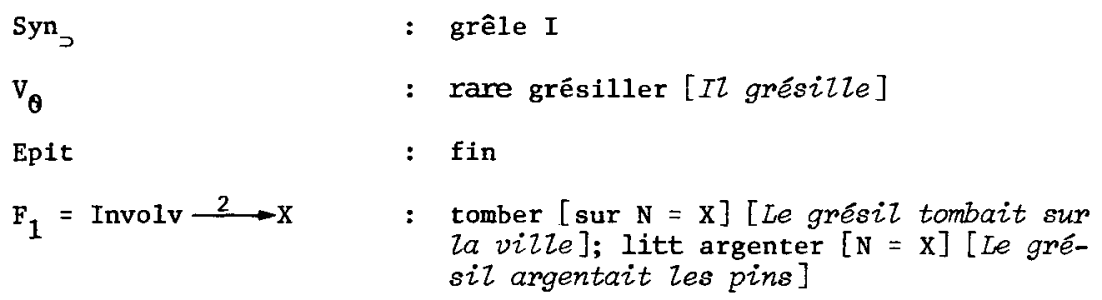
la vizle]; litt argenter [N = x] [Le grésil argentait les pins]

un être $\mathrm{X}$

$\leftarrow$\begin{tabular}{l}
1 \\
\hdashline CausnonConv \\
21
\end{tabular} $\mathrm{~F}_{1}: \begin{aligned} & \text { s'abriter, se cacher, se protéger } \\
& {[\text { de ART } \sim]}\end{aligned}$

3. FIN III.1

'dont les quanta sont très petits' [sable ssel> fin, poudre $\langle$ pluie fine]. 


\section{EXEMPLES}

La brise piquait, et un fin grésil, glissant sur les vêtements sans les mouiller, gardait fidèlement la tradition des Noëls blancs de neige [A. Daudet]. Ce n'est pas de la neige qui tombe, c'est du grêsil. Les grains de grésil sont parfois couverts d'une couche de glace transparente.

GRÊLON, nom , masc.

Grain de glace qui tombe des nuages I sur la terre en tant que phénomène atmosphêrique $[$ = Sing (grête) $]$.

\section{FONCTIONS LEXICALES}

$\begin{array}{ll}\text { Func }_{\emptyset} & : \text { [il] tomber [des } \sim s] \\ \text { Func }_{0} & : \text { tomber } \\ {\text { Magn }\left(\text { Func }_{0}\right)} & : \text { abondamment, dru, fort } \\ & \text { EXEMPLFS }\end{array}$

Les grêlons étaient poussés par un grand vent. Il tombe de gros grêlons. Elle ne remarqua pas que les gouttes de pluie étaient presque aussi dures que des grêlons.

3. Problèmes de la description sémantique des lexèmes dans le DEC

3.1 Composante sémantique conmine à tous les éléments d'un champ sémantique 4 .

4. Nous distinguons, dans notre usage, champ lexical et champ sémantique.

'Un champ sémantique' (concept de base) est 1 'ensemble des lexèmes dont les définitions ont une composante commune NON TRI- 
Le DEC inclut dans ses dëfinitions des lexèmes appartenant au même champ sémantique, une composante qui exprime cette appartenance de façon explicite. Dans ce cas concret, c'est 'phénomène atmosphérique' - composante que $1^{\prime}$ 'on retrouve dans PLUIE I, NEIGE et GRÊLE I, c'est-à-dire dans les trois lexèmes "atmosphériques" de base. (Les définitions respectives de leurs dérivés sémantiques PLEUVOIR I, NEIGER, GRÊLER et GRÉSIL ne contiennent cette composante que de façon implicite, c'est-à-dire par l'inclusion des lexèmes de base.) La présence d'une telle composante commune permet de formuler des règles plus générales concernant le champ sémantique donné et d'introduire des abréviations et généralisations utiles: par exemple, on pourrait rêunir certaines informations lexicographiques communes à (presque) tous les ëléments du champ sémantique, dans 1'article de dictionnaire affecté à 1 'entrée qui correspond à cette composante commune. Dans notre cas, toutes les généralisations qu'on voudrait énoncer à propos des 'phénomènes atmosphériques' pourraient être emmagasinées sous PHÉNOMĖNE ATMOSPHÉRIQUE, sans être répétées à plusieurs reprises dans les articles de lexèmes particuliers.

VIALE. (Nous ne sommes pas capables de préciser ce caractère non trivial des composantes dont il est question. Nous pouvons seulement dire qu'une composante sémantique doit être intuitivement acceptable comme telle.) Par exemple, PLUIE I, NEIGE, GRÊLE I, PLEUVOIR I, etc. appartiennent au champ sẻmantique 'phénomène atmosphérique'.

'Un champ lexical' est l'ensemble des vocables dont les lexèmes clés (ou lexèmes de base) appartiennent au même champ sëmantique. Par exemple, tous les vocables présentés ici forment un champ lexical parce que tous leurs lexèmes de base appartiennent au même champ sémantique 'phénomène atmosphérique'. 
Dans un deuxième temps, 1'usage de composantes sémantiques communes à un champ sémantique aide à dégager des schémas généralisếs des définitions pour les lexèmes de ce champ, ce qui contribue à la standardisation et à l'homogénéité des définitions: tous les instruments, toutes les parties du corps, tous les déplacements, etc. seront décrits suivant un schéma général préétabli. Ce procédé constitue un pas important vers le développement de la GRAMMAIRE DU DICTIONNAIRE, un but aussi ambitieux qu'important pour la lexicographie.

\subsection{Définition lexicographique et cooccurrence lexicale libre}

Considérons les exemples suivants:

(1) a. une pluie de courte durée; pendant la pluie; Les trois pluies [de la semaine dernière...]

b. *une neige de courte durée; *pendant la neige; *Les trois neiges [de la semaine dernière...]

c. ?une grêle de courte durée; *pendant la grêle; *Les trois grêles [de la semaine dernière...]

$$
\left.\mid \begin{array}{ll}
\text { La } & \text { pluie } \\
\text { La } & \text { grêle } \\
\text { La } & \text { neige }
\end{array}\right\} \text { fouettait les fenêtres }
$$

$$
\text { On voit encore }\left|\begin{array}{rll}
\text { *de la } & \text { pluie } \\
\text { de la } & \text { neige } \\
\text { de la grêle }
\end{array}\right| \begin{aligned}
& \text { sur 1'asphalte de } \\
& \text { la piste }
\end{aligned}
$$

I1 en découle trois conclusions sémantiques importantes:

- 'La pluie' est un événement qui peut avoir une durée, être localisé dans le temps et qu'on peut compter; 'la neige' et 'la grêle' ne sont pas des événements.

- 'La pluie' est, en même temps, une substance (= 1'eau), puisque c'est la substance qui fouette les fenêtres; ' la neige' et ' la grêle' sont aussi des substances. 
- Mais 'la pluie' est une substance que l'on ne peut pas voir au repos, à la différence de '1a neige' et de 'la grêle': la pluie peut tomber, tambouriner, cingler..., alors qu'elle ne peut pas *couvrir, *remplir [au sens statique!]. *être vue sur des objets...

Ces faits nous amènent à construire nos définitions exactement de 1a façon dont nous $l^{\prime}$ avons fait: PLUIE $I='(\alpha)$ chute d'eau... I = événement $]$ ou $(\beta)$ cette eau $[=$ substance $]$ qui tombe $[=$ de façon active ]'; NEIGE/GRÊLE $I={ }^{\prime}(\alpha)$ substance... ou $(\beta)$ la chute de cette substance...|... seulement avec...'. La composition et la structure d'une définition doit assurer la cooccurrence lexicale libre correcte du lexème correspondant (la cooccurrence restreinte étant couverte par les fonctions lexicales): ainsi, nos définitions interdiront des cooccurrences libres du type ttrois neiges 〈grêles>, tune neige 〈grêle> contimuelle car une 'substance' ne peut être comptée ou avoir une durée tandis que trois pluies, une pluie continuelle pourront être engendrés par nos définitions car une 'chute' peut être comptée et avoir une durée.

Prenons maintenant les quatre cas suivants qui semblent présenter des difficultés pour les définitions proposées.
A) On peut dire (4):

(4)

$$
\text { La neige }\left\{\begin{array}{l}
\text { a cessé } \\
\text { a commencé à trois heures } \\
\text { continue toujours }
\end{array}\right\}
$$

Ici la neige est sans doute interprêtêe comme 'chute de neige '; c'est pourquoi nous avons inclus la composante 'chute... [ = événement]' dans la définition de NEIGE. Pourtant 11 y a une restriction: ce sens de 
NEIGE n'est possible qu'avec quelques verbes que nous avons distingués; on ne peut pas 1'actualiser de façon libre, comme on le peut avec PLUIE I:

(5) $\left\{\begin{array}{c}\text { une pluie } \\ \text { *une neige }\end{array}\right\}$ sans fin, $\left\{\begin{array}{c}\text { une pluie } \\ \text { *une neige }\end{array}\right\} \begin{aligned} & \text { de trois } \\ & \text { heures, } \ldots\end{aligned}$ (On a d'autres exemples en (1). Pour NEIGE, la composante 'chute...' est phraséologiquement liée, c'est-à-dire qu'elle n'est manifestée qu'avec quelques FL inventoriêes dans le dictionnaire comme par exemple La neige a commencé; nous la considérons comme relevant plutôt đe I'expression entière. Par conséquent, les expressions tout à fait correctes sont décrites par les fonctions lexicales dans l'article NEIGE. Le lexème GRÊLE I se comporte et, par conséquent, est décrit de la même façon: les FL IncepFunc ${ }_{0}$, FinFunc ${ }_{0}$ et ContFunc ${ }_{0}$ indiquent la possibilité de dire la grêle a commencé à trois heures <a cessé; continue toujourss. Pourtant la condition imposée à la composante 'chute...' dans les définitions de NEIGE et de GRÊLE I exclut les expressions libres du type *trois neiges <grêles>, *la dernière neige 〈grêlè, *une neige 〈grểe contimuelle, etc.

B) Les phrases (6) sont pourtant bonnes:

$$
\text { Après }\left\{\begin{array}{ll}
\text { la neige } \\
\text { la grêle }
\end{array}\right\} \text {, tout semble changé }
$$

Un contre-exemple? Pas du tout: la préposition après - à la différence, par exemple, de pendant - accepte facilement les noms a'objets et de substance s'il est possible de les interpréter:

(7) a. après le vin; après moi, Jean est venu; après ce roman 
(7) b. *pendant le vin; *pendant moi; *pendant ce roman

Tout simplement, après ne peut pas servir de pterre de touche pour tester la présence de la composante 'événement/action/procès'. Pour cela, il faudra utiliser pendant, et pendant n'accepte ni neige, ni grềle (voir supra).

C) On peut aussi très bien dire (8):

$$
\text { La météo a annoncé }\left\{\begin{array}{ll}
\text { de la neige } \\
\text { de la grêle }
\end{array}\right\} \text { pour ce soir }
$$

I1 est évident qu'en français on n'annonce de façon libre que des événements:

(9) a. On a annoncé le mariage de Jean et Marie <la mort du ministre, 1'arrivée de l'expédition, ...>

b. *0n a annoncé le président Reagan sde la soupe aux oignons, un nouvel ordinateur, ce véhicule tout-terrain, ...>

(Nous avons ici en tête le même sens d'ANNONCER que nous voyons dans (8): 'indiquer comme devant se produire prochainement'.)

Pourtant, le verbe ANNONCER dans le sens considéré est couramment employé avec les compléments d'objet désignant un phénomène atmosphérique, qui ne sont pas toujours des événements: annoncer du beau temps <des vents, du brouizlard, du soleil, ...>; l'article partitif dans ces locutions exclut 1'interprétation des termes atmosphériques cités comme événements ponctuels. Ceci nous fait postuler un sens particulier d'ANNONCER: phérique $\mathrm{Y}$ va avoir lieu' 
(avec le schêma de régime spécifiant que le complément d'objet $[=\mathrm{Y}]$ doit être muni de l'article partitif).

Nous proposons donc que ce nouveau sens, c'est-à-dire un nouveau lexème ANNONCER, soit inscrit dans le dictionnaire. Remarquons que ce verbe tend à avoir des sens phrasêologiquement restreints:

- annoncer un film <une pièce de thêâtre>;

- annoncer une tierce <deux sans atout>, dans des jeux de cartes;

- annoncer quelqu'un à Madame [un domestique]

Par conséquent, en y ajoutant un sens de plus, nous ne violons pas trop, semble-t-i1, la nature du verbe ANNONCER.

La leçon à en tirer est qu'en observant la combinatoire libre d'un lexème en vue d'en déterminer la structure sémantique, il faut faire attention à la description de ses cooccurrents.

D) Le lexème NEIGE peut avoìr un pluriel:

(11) les neiges éternelles du sommet, les neiges arctiques, les neiges sans fin et sans limite ...

Est-ce que ce fait ne contredit pas notre définition de NEIGE comme 'substance'? Une substance, en principe, ne peut pas être comptée. La réponse est évidente: ici, le sens ou le contenu sémantıque du pluriel morphologique n'est pas la pluralité numérique. Le pluriel signifie, auprès de NEIGE, une grande quantité et produit un certain effet artistique; comparez les eaux, les flots, les sables, ... Pour tester le caractère "comptable"/"non comptable" d'un lexème, il faut 1e combiner avec des numéraux, et ce dernier test démontre immêdiatement que 
'la neige' et 'la grêle' ne sont pas comptables (*trois neiges, *trois grềles).

\subsection{Disjonction dans les définitions}

L'emploi de l'élément sémantique 'ou' dans les définitions pose le problème suivant: quand faut-il admettre une disjonction des composantes sémantiques à l'intérieur d'une même définition et quand estil préférable d'en faire deux définitions? C'est-à-dire, un lexème L

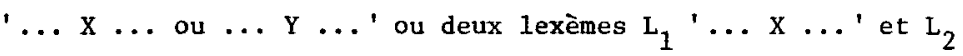
'... Y ...'? L'économie de la description milite pour la première solution, la simplicité logique favorise la deuxième. Sans essayer de formuler une solution théorique globale, nous nous limiterons à deux recommandations pratiques.

Considérons d'abord le cas le plus simple de PLUIE II.2 (une pluie de fleurs) et GRELE II (une grểe de pierres). Pour tester I'emploi de la disjonction dans les dëfinitions, nous n'analyserons que PLUIE II.2, étant donné que les deux lexèmes en question sont des quasi-synonymes (PLUIE II.2 ᄀ GREELE II). (On remarquera une différence importante qui n'est, cependant, pas pertinente dans le cadre présent: dans grêle de $X$, les $\mathrm{X}$ nuisent à $\mathrm{Y}$, de sorte qu'on a grêle de balles <d'injures>, mais non *grêle de fleurs <de compliments>; pluie est neutre sous ce rapport: pluie de balles sde fleurss, pluie d'injures <de compliments>. Nous $\mathrm{n}^{\prime}$ insisterons pas sur cette distinction, qui est reflétée, dans la définition de GRÉLE II, par la composante' '... tel que les $\mathrm{X}$ sont nuisibles à $\mathrm{Y}$....'.)

Le complément $[=\mathrm{x}]$ introduit par la préposition de désigne des 
objets tombant ou étant projetés sur Y ou des actes (verbaux ou physiques) concernant Y: pluie de balles - pluie de coups. Mais ce choix n'affecte en rien les propriétés syntaxiques, lexico-combinatoires ou morphologiques du lexème PLUIE II.2; pluie de balles et pluie de coups sont employés strictement de la même façon. Les membres de la disjonction n'ont pas de corrêlats dans les autres zones de 1'article de dictionnaire. Cependant, dans la zone des FL de l'article GRÊLE II, de tels corrélats sont présents mais en si petit nombre (2) que nous avons quand même formulé la définition sous la forme d'une disjonction. Le fait que des corrélats apparaissent dans la zone des FL est un argument moins décisif que s'ils apparaissent dans les zones morphologiques et syntaxiques car les cooccurrences lexicales sont beaucoup moins systêmatiques que les traits morphologiques et syntaxiques. D'où notre première recommandation:

Si la disjonction ' ... X ... ou ... Y ...' ne correspond à aucune distinction dans les autres zones de la description lexicographique, il est préférable de la garder au sein d'une même définition.

Suivant la recommandation, nous préférons préserver l'unité des définitions de PLUIE II.2 et GRÊLE II.

De surcroît, il est possible de dire (12):

(12) a. une pluie de fleurs et de compliments

b. une grêle de pierres et d'injures

c'est-à-dire qu'il est possible de rêunir au moyen de la conjonction des objets et des actes auprès de $1 \mathrm{a}$ même occurrence du lexème analysé. Ceci signifie qu'il faut préserver 1'emploi de la disjonction 
dans les définitions des lexèmes PLUIE II.2 et GRÊLE II: en effet, si on avait deux lexèmes différents PLUIE II.2' (= pluie de fleurs) et PLUIE II.2" (= pluie de compliments), lequel des deux serait représenté dans (12a)? Et (12a) provoquerait un calembour. Par conséquent, la solution voulant que la disjonction "objets ou actes" reste à 1 'intérieur de la même définition, devient obligatoire, dans ce cas. Nous résumerons ce qui vient d'être dit sous la forme d'une deuxième recommandation:

Si un lexème du type $\mathrm{L}$ ' $\ldots$ X . .. ou ... Y ...' admet, au niveau de la syntaxe, la conjonction syntaxique de ses deux compléments $L_{1}$ '...X X...' et $\mathrm{L}_{2}, \ldots \mathrm{Y} \ldots$ ', de sorte qu'on $\mathrm{a}^{1}$

$$
\begin{aligned}
& \mathrm{L} \stackrel{\text { SYNT }}{\longrightarrow} \mathrm{I}_{1} \text { et } \mathrm{L}_{2} \\
& \text { (ou } \mathrm{L} \stackrel{\text { SYNT }}{\longrightarrow} \mathrm{L}_{2} \text { et } \mathrm{L}_{1} \text { ) }
\end{aligned}
$$

alors la disjonction sémantique doit rester au sein d'une même définition (même si cette disjonction a des corrélats dans les autres zones).

I1 s'agit 1à, en fait, du soi-disant CRITĖRE D'APRESJAN. Appliqué au lexème PLUIE I [phénomène atmosphérique], il donne le résultat suivant. PLUIE I est ' 1 a chute d'eau... [ = événement] ou 1 'eau qui tombe... [= substance]'. Cette disjonction affiche plusieurs corrélats morphologiques et combinatoires:

- Le pluriel n'est possible que pour le premier membre de la disjonction.

- Les expressions rideau <mur> de pluie actualisent le deuxième membre de la disjonction; les épithètes fine ou memue se réfèrent au deuxième membre $[=\mathrm{eau}]$, tandis que l'épithète continuelle et l'attribut sans trêve se réfèrent au premier membre [= chute]. 
Quand on dit La pluie l'a squcée, on parle de l'eau, mais en disant une pluie soudaine, on désigne la chute (d'eau); et ainst de suite.

Néanmoins, on peut dire (13):

(13) a. Une soudaine pluie l'a tout trempé

[soudaine actualise 'chute', et tremper, ' 1 'eau'].

b. La pluie glaciale, qui avait commencé au petit jour, tambourinait sur leur tente

[commencer actualise 'chute', et glaciale et tambouriner, '1'eau'].

D'autres exemples de ce type abondent. Par conséquent, suivant le critère d'Apresjan, nous devons garder la disjonction 'chute... ou i... eau' au sein d'un même lexème.

\subsection{GRELLE I et GRÊLON}

Au premier abord, il semble que la grêle I soit - très grossièrement: - 'de la glace qui tombe en grains des nuages I'; c'est ainsi que le lexème est couramment défini dans les dictionnaires. Mais alors un grêzon est un 'grain de glace qui tombe des nuages I'. Cependant, le DEC suit le Principe du Bloc Maximum:

Si une définition lexicographique inclut une expression multilexémique $\mathrm{L}_{1}+\mathrm{L}_{2}+\ldots+\mathrm{L}_{\mathrm{n}}$ sémantiquement équivalente à un seul lexème $L$, il faut remplacer $\mathrm{L}_{1}+\mathrm{L}_{2}+\ldots+\mathrm{L}_{\mathrm{n}}$ par $\mathrm{L}$.

Le lexème $\mathrm{L}$ est le "Bloc Maximum" évoqué ci-dessus. Nous n'allons pas expliquer et justifier ce principe ici mais $1^{\prime}$ 'accepter tout simplement comme postulat.

Dans la définition de GRÊLE I, nous avons 'de la glace qui tombe en grains des nuages $I$ ' mais 'grains de glace qui tombent des nuages $I$ ' 
sont des GRÊLONS. Le Principe du Bloc Maximum nous force à effectuer le remplacement prescrit, ce qui nous amène à la dẻfinition formulée: GRÊLE I $\cong$ 'substance composée de grêlons'.

Il importe d'insister sur le fait que la relation sémantique entre le nom d'une substance et le nom d'un quantum de cette substance n'est pas toujours la même. Prenons deux exemples:

A) NEIGE $\not$ 'substance formêe de flocons', pour la raison évidente voulant que des flocons ne sont pas nécessairement de neige.

B) GLACE I.I $\not$ 'substance composée de glaçons', puisque, si on avait une telle définition, on devrait définir GLAÇon comme 'morceau d'une substance solide, très froide et transparente formée d'eau congelée', et suivant le Principe du Bloc Maximum, on devrait remplacer 'substance solide, ... d'eau congelée' par GLACE I.1, ce qui force les définitions GLACE $1.1 \cong$ 'substance solide, très froide et transparente formée d'eau congelée' et GLAÇON = 'morceau de glace I.1'. À cause de la nature différente des quanta, les formulations des définitions diffèrent les unes des autres.

3.5 Les noms et les verbes dans le domaine "atmosphérique"

En français, les noms "atmosphériques" sont sêmantiquement primaires par rapport aux verbes correspondants (ceci est en accord avec tous les dictionnaires courants).

En effet, nous ne pouvons définir PLUIE I comme 'action $\left[=\mathrm{s}_{0}\right]$ de pleuvoir I' puisque PLUIE I signifie 1a chute d'eau ET 1'eau tombante. On ne peut pas non plus dire que PLUIE I est 'l'eau qui pleut $I$ ', puisque alors PLEUVOIR $I=$ 'de 1 'eau tombe des nuages $I$ en tant 
que phénomène atmosphérique' et que la substitution nous donne immédiatement ' 1 'eau qui tombe ... atmosphêrique'; nous yoyons donc que 'pluie I' est primaire.

NEIGE ne peut pas être dêfinie en termes de 'neiger' puisqu'il existe de la neige (dans les montagnes, dans les régions polaires, etc.) qui n'est pas 'résultat de neiger' et qui ne neige pas. NEIGER implique la NEIGE mais pas inversement; par conséquent, 'neiger' est sémantiquement plus complexe.

GRÊLER I veut dire qu' 'il tombe des grêlons'; mais puisque 'ensemble [ = substance composée] de grêlons' = GRÊLE I, grểer I doit être défini en termes de grêle $I$. Il est, par contre, impossible de définir GRÊLON en termes de GRELLER I.

\author{
Suzanne Mantha et \\ Igor Mel'ouk \\ Université de Montréal
}

\title{
Remerciements
}

Lors de cette recherche, nous avons grandement profité de la collaboration de Lidija Iordanskaja. Les remarques et suggestions de L. Elnitsky nous ont beaucoup aidés à donner à notre description sa forme finale. Le texte définitif a été lu par N. Arbatchewsky-Jumarie, J. Labelle et Y.-C. Morin qui nous ont communiqué leurs remarques. Les lecteurs du comité de sélection de la Revrre québêcoise de linguistique nous ont aussi suggéré des modifications. Nous exprimons ici notre gratitude à tous ces collègues, bien que nous restions seuls responsables des erreurs possibles et des solutions proposées. 


\author{
RÉFÉRENCES
}

ARBATCHEWSKY-JUMARIE, $N$. et L. IORDANSKAJA (1982) "Lexicographic App1ications of Lexical Functions: two sample lexical entries from an Explanatory-Combinatorial Dictionary", Proceedings of the Eighth Annual Meeting of Berkeley Linguistics Society, Berkeley, UCB, p. 364-372.

ARBATCHEWSKY-JUMARIE, $N$. et L. IORDANSKAJA (à paraître) "'Parties du corps' dans le Dictionnaire explicatif et combinatoire du français contemporain: sémantique et structure des vocables", Cahiers de lexicologie.

COHEN, B. et L. ELNITSKY (à paraître) "Un nouveau type de description lexico-sémantique utile au traducteur. Un exemple: le mot PRIX", Méta.

DAGENAIS, L. (à paraître) "Le problème de la description sémantique en lexicographie (les vocables français PERMETTRE, DÉFENDRE, AUTORISER, INTERDIRE)", Cahiers de lexicologie.

ELNITSKY, L. (1982-I) "Une description du verbe FLAMBER: exercice dictionnairique, Cahiers de lexicologie, vol. 40, no 1, p. 95-111.

MEL'CUK, I.A. (1974) Opyt teorii lingvističeskix modelej "Smysl Tekst",[Essai d'une théorie des modèles 1inguistiques du type "Sens $\longrightarrow$-Texte"] (traduction du titre), Moscou : Nauka.

MEL'CUK, I.A. (1982) "Lexical Functions in Lexicographic Description", Proceedings of the Eighth Annual Meeting of the Berkeley Linguistics Society, Berkeley, UCB, p. 427-444.

MEL ' CUK, I.A., N. ARBATCHEWSKY-JUMARIE et L. IORDANSKAJA (1981) "Un nouveau type de dictionnaire: le Dictionnaire explicatif et combinatoire du français contemporain (six articles de dictionnaire)", Cahiers de lexicologie, vol. $38, n^{\circ} 1$, p. 3-34.

MEL 'CUK, I.A., N. ARBATCHEWSKY-JUMARIE, L. IORDANSKAJA et A. LESSARD (1983) "Trois principes de description sémantique d'une unité lexicale dans un Dictionnaire explicatif et combinatoire", Revue Canadienne de Linguistique, vol. 28, no 2, p. 105-121.

MEL'CUK, I.A. et al. (à paraître) "Vers un Dictionnaire explicatif et combinatoire du français contemporain (études lexico-sémantiques)", Presses de 1'Université de Montréal. 